\title{
Adsorption structure and scanning tunneling data of a prototype organic-inorganic interface: PTCDA on Ag(111)
}

\author{
Michael Rohlfing, ${ }^{1, *}$ Ruslan Temirov, ${ }^{2}$ and Frank Stefan Tautz ${ }^{2}$ \\ ${ }^{1}$ Fachbereich Physik, Universität Osnabrück, Barbarastraße 7, 49069 Osnabrück, Germany \\ ${ }^{2}$ School of Engineering and Science, Jacobs University, P.O. Box 750 561, 28725 Bremen, Germany
}

(Received 7 June 2007; published 17 September 2007)

\begin{abstract}
We present density-functional calculations of a monolayer of 3,4,9,10-perylene-tetracarboxylic-dianhydride adsorbed on the $\operatorname{Ag}(111)$ surface, yielding detailed insight into the structural and electronic properties of this prototypical adsorption system. Using the local-density approximation as the best choice for the exchangecorrelation functional, we discuss the bond lengths inside the molecules, the distortion of the molecules due to adsorption, and their position and orientation relative to the substrate and to each other. Based on the calculated geometric and electronic structures, we calculate scanning tunneling microscopy and spectroscopy data within the Tersoff-Hamann framework [Phys. Rev. B. 31, 805 (1985)]. To this end, two-dimensional Fourier transform methods and spatial extrapolation techniques are employed to evaluate the sample wave functions at the tip position. We obtain constant-current images and spectral data that reveal detailed information about the electronic structure of the system. In addition, we have measured the same data by low-temperature scanning tunneling microscopy and scanning tunneling spectroscopy. Our measured and calculated data are in good agreement with one another.
\end{abstract}

DOI: 10.1103/PhysRevB.76.115421

PACS number(s): 68.43.Fg, 73.20.Hb, 68.43.Bc, 68.37.Ef

\section{INTRODUCTION}

The adsorption of $\pi$-conjugated organic molecules on metal substrates constitutes a fast-growing field, motivated both by fundamental questions and by prospective applications in organic and molecular electronics. One prototypical example is provided by 3,4,9,10-perylene-tetracarboxylicdianhydride (PTCDA) on silver surfaces, which belongs to the best-studied systems of large aromatic molecules on noble metals. ${ }^{1-24}$ The adsorption of PTCDA on the $\mathrm{Ag}(111)$ surface has attracted particular attention because it results in a commensurate monolayer structure. ${ }^{4,5,7,13}$ Due to the high quality of available data for this system, obtained by a wide range of surface analytical techniques, it is an ideal test case to address general questions of the interaction between large $\pi$-conjugated molecules and metal surfaces.

In a combined experimental and theoretical effort, ${ }^{15,18,20}$ we have investigated the geometric and electronic structures of the PTCDA:Ag(111) adsorption system. The purpose of the present paper is to discuss the theoretical aspects of this work in detail and to explore their relation to the experimental results more systematically.

Although a lot of work has already been devoted to the theoretical understanding of the PTCDA:Ag(111) system, ${ }^{10,15,17,18,20,23}$ it still constitutes a big challenge to theory for two reasons. On the one hand, this is due to the high numerical demand of carrying out calculations for systems of this size and complexity. On the other hand, a rather inconsistent picture has emerged from several calculations reported recently for the PTCDA:Ag(111) system, ${ }^{10,15,17,18,20,23}$ leaving a number of important issues concerning the molecular structure and bonding mechanism unresolved. This unsatisfactory situation can be ascribed to fundamental problems and limitations of density-functional theory (DFT) when applied to the moderately strong adsorption of extended aromatic molecules on (noble) metal sur- faces. Against this background, we present here a comprehensive theoretical analysis of PTCDA on $\operatorname{Ag}(111)$, including adsorption sites and orientations, bond lengths, molecular distortions, electronic structure and its site specificity, charge transfer and substrate-bonding mechanisms, intermolecular interactions, and scanning tunneling microscopy (STM) image contrast and spectroscopy. All these issues are discussed within close conjunction with experimental results.

One of the most important issues is the origin of the binding to the substrate. Two different chemisorption mechanisms have been suggested, ${ }^{10,15,17,18,20,23}$ i.e., binding via electronic charge transfer from the metal into the molecule and binding between the carboxylic oxygen atoms and substrate silver atoms. The two chemisorption mechanisms between PTCDA and $\operatorname{Ag}(111)$, which have already been investigated by us before, ${ }^{15,18,20}$ are controversially discussed in the literature. ${ }^{10,17,23}$ The first of these mechanisms involves transfer of electronic charge from the metallic substrate into the lowest unoccupied molecular orbital (LUMO) of the molecule. Due to the wide spatial extent of the LUMO, the entire molecule participates in this form of binding, which can thus be considered as rather delocalized. Such binding would be quite insensitive to local details of the atomic structure of the molecule or the substrate, like steps or point defects. In some calculations of the PTCDA: $\operatorname{Ag}(111)$ adsorption system, a significant charge transfer (and corresponding nonlocal binding) is observed, ${ }^{10,15,18}$ while others find no charge transfer. ${ }^{23}$ The second mechanism via the oxygen atoms, on the other hand, is of a strongly localized nature and would depend sensitively on the precise atomic position, on the occurrence of defects, and on modifications of the molecular end groups. Such local binding is characterized by local redistribution of the electronic charge at the oxygen atoms (in particular, the carboxylic ones), combined with the attraction of these atoms to the substrate and local deformation of the molecule, which is flat in its gas-phase configu- 
ration. In the calculations reported up to now, the vertical deformation and bending of the molecule range from $\sim 0$ (Refs. 10 and 17) to $\sim 0.5 \AA{ }^{23}$ which poses the question of whether local bonds as described above are important for the binding of the molecule or not.

Most experiments and theoretical calculations, including our own studies, indicate that both chemisorption mechanisms are present in the binding of PTCDA to $\operatorname{Ag}(111)$. On the experimental side, charge transfer can be concluded from photoemission spectroscopy, ${ }^{1,21}$ scanning tunneling spectroscopy, ${ }^{5,20}$ and vibrational spectroscopy, ${ }^{8,9,11,14}$ thus confirming that the first binding mechanism is present. Furthermore, normal incidence $\mathrm{x}$-ray standing wave (NIXSW) measurements indicate a significant deformation of the molecule with a vertical distortion of approximately $0.3 \AA$ for the oxygen atoms at the molecular end groups. ${ }^{15,24}$ These deformations indicate that local binding of the oxygen atoms to the substrate takes place, in addition to the nonlocal binding via occupation of the LUMO. Both observations are consistent with our findings within density-functional theory, which are discussed in detail in Sec. II B. In particular, we show in this paper that the two binding mechanisms support each other rather than compete with one another.

In addition to the two chemisorption mechanisms, longrange van der Waals interaction ${ }^{25-27}$ is most certainly relevant for the present system as well, constituting a third interaction mechanism. van der Waals interaction results from electronic polarizability and contributes to the electronic correlation energy in a nonlocal way; it is not accurately described within the DFT exchange-correlation functionals commonly employed today, although significant progress has already been achieved to implement van der Waals mechanisms into the DFT framework. ${ }^{28-30}$ Consequently, the asymptotic behavior of the binding energy at large substrateadsorbate distance results wrongly from DFT calculations, and the binding at equilibrium distance can be overestimated or underestimated. In our present work, however, we assume that the vertical energetics of the adsorption process is given by DFT in the local-density approximation (LDA) with sufficient accuracy (see below).

Of particular interest are the adsorption height and binding energy of the entire molecule, because both are measures of the absolute metal/molecule interaction strength. While experiments indicate an adsorption height of about $2.9 \AA,{ }^{15,24}$ the results obtained from theory scatter significantly. ${ }^{10,15,17,18,20,23}$ One important reason for this variance is the problem of DFT in describing van der Waals interaction, which is a significant contribution to the energetics of the present case, despite the fact that the PTCDA: $\operatorname{Ag}(111)$ interaction clearly is of chemical nature. ${ }^{1,2,11,14,21}$ The $\mathrm{LDA}^{31}$ results in a PTCDA:Ag(111) adsorption distance of about $2.8 \AA{ }^{10}$ which we find in our present work as well, combined with an adsorption energy of about $3 \mathrm{eV}$ per molecule (see Sec. II B). Within the generalized gradient approximation (GGA), the Perdew-BurkeErnzerhof functional ${ }^{32}$ (GGA-PBE) yields a height of about $3.5 \AA$ (Refs. 17 and 18) and an adsorption energy of less that $0.1 \mathrm{eV}$. The Perdew-Wang GGA functional ${ }^{33}$ (GGA-PW91) finally results in an adsorption height of about $3.2 \AA$ and an adsorption energy of about $0.5 \mathrm{eV}$, accompanied by strong bending of the molecule. ${ }^{23}$
While the failures of DFT in describing van der Waals interaction constitute an unsatisfactory situation, we will, nevertheless, take the LDA as the best approach available at the moment within the framework of DFT. In contrast to quantum chemical cluster calculations, see, e.g., Ref. 34, which treat electron correlations more systematically, we are, therefore, able to describe a fully periodic system in which structure-specific molecule-molecule interactions are also taken into account. This latter point is important because, in addition to the molecule-substrate interaction, these intermolecular interactions play an important role for the geometric and electronic structures of PTCDA on $\operatorname{Ag}(111)$, as we will show below. The resulting DFT geometry is in good agreement with the available experimental data, in particular, with the overall adsorption distance and the local distortion of the molecule (see Sec. II B). This allows us to propose a detailed structure model of the interface, also covering aspects which are inaccessible to experiment, to identify subtleties of the two bonding mechanisms discussed in Refs. 15 and 18, and to provide input for further investigations in the framework of density-functional theory.

A second topic of the current work is STM in the case of PTCDA: $\operatorname{Ag}(111)$. STM and scanning tunneling spectroscopy (STS) are powerful approaches to the local geometric and electronic structures on the atomic length scale. In STMSTS, the electronic structure and its relation to the geometric structure can be directly observed. As a counterpart to experiment, theoretical calculations are important for rationalizing the imaging process and for deriving a microscopic model of the adsorption geometry and the system's electronic structure. Such calculations can be carried out if the electronic wave functions and energies are known. ${ }^{35-40} \mathrm{It}$ must be noted, however, that STM experiments display electronic states, seldomly reaching a lateral spatial resolution of better than about $1 \AA$. In order to identify atomic positions with subangstrom resolution in STM images, the best approach is, in general, to calculate the STM images resulting from a structural model and to compare them with experiment. By such a comparison, it was possible to identify the adsorption sites of the PTCDA monolayer on $\operatorname{Ag}(111)$ as bridge positions with an accuracy of $0.2 \AA .{ }^{20}$ In addition, by analyzing the spatial and energetic features of the tunneling experiment, one can identify the electronic states and their occupation status. One important observation in this context concerns the partial occupation of the LUMO of free PTCDA when the molecule adsorbs on the surface, thus indicating that significant charge transfer from the substrate to the molecule takes place. ${ }^{5,20}$

The discussion of STM images and STS spectra in this paper is based on the structures calculated in the framework of DFT. Due to limited knowledge about the tip, we restrict ourselves to the Tersoff-Hamann approach in evaluating the tunneling current. ${ }^{35-39}$ While this approach does not easily permit the evaluation of absolute tunneling currents, it does allow one to identify important structural and spectroscopic features for many systems, including transition-metal surfaces and adsorbates. In the present case, the images which we obtain show a large variety of different features, depending on the nature of the relevant tip orbital, tunneling voltage, and sample-tip distance, in good overall agreement with experimental data. 
The paper is organized as follows. In Sec. II, we discuss in detail the adsorption geometry of a monolayer of PTCDA on $\mathrm{Ag}(111)$. In Sec. III, we outline our approach to calculating scanning tunneling data on the basis of densityfunctional calculations. The resulting STM and STS data of the current system are discussed in Sec. IV. A brief summary concludes the paper in Sec. V.

\section{ADSORPTION GEOMETRY}

The geometric structure of a monolayer of PTCDA on $\operatorname{Ag}(111)$ is studied within the framework of densityfunctional theory. Before discussing the results, the method is briefly outlined.

\section{A. Computational approach: Density-functional theory}

Our calculations of both the structure and the scanning tunneling data presented in Sec. IV are based on conventional DFT for the electronic ground state, employing LDA. In the present context of weakly binding adsorbates on noble-metal surfaces, the role of the exchange-correlation functional is a difficult issue. The LDA yields too strong adsorption energies and too short adsorption distances. The GGA available so far result in too weak adsorption and too large adsorption distances. Here, the problems of the functional of $\mathrm{PBE}^{32}$ seem to be even more dramatic ${ }^{17,18}$ than those of the functional of Perdew and Wang, ${ }^{33}$ which does yield adsorption of reasonable strength. ${ }^{23}$

None of the functionals (LDA or GGA) describes the long-range van der Waals attraction between the adsorbate and the substrate correctly. Since this van der Waals interaction significantly contributes to the correlation energy of the system and certainly plays an important role for the adsorption of PTCDA to $\operatorname{Ag}(111)$, one might argue that within the approximations available today, DFT is not the fully appropriate approach for the present system. Fortunately, however, it turns out that DFT does yield the correct lateral structure of PTCDA on $\operatorname{Ag}(111),{ }^{20}$ and that it also yields the correct trends concerning the internal distortion of the molecule upon adsorption. ${ }^{15,18}$ The adsorption sites come out correctly because van der Waals interaction tends to be site unspecific. It results from the perturbative interaction of electronic polarizability inside the molecule and inside the substrate, which generates long-range, nonlocal contributions to the correlation energy of the system. In contrast to local bonds, these effects vary rather weakly with respect to position; in particular, they are quite insensitive to the lateral position of the molecule on the substrate. The problems of using DFT for van-der-Waals-dominated adsorption systems refer mainly to the absolute vertical position of the molecule as a whole, which is, indeed, not correctly given by our DFT calculations (see below). In particular, the asymptotic behavior $\left(\sim 1 / Z^{3}\right)$ of the binding energy, depending on the substrate-adsorbate distance $Z$, is different from that given by DFT $\left(\sim e^{-\lambda Z}\right)$, and at equilibrium height, the DFT energy may lead to underbinding (in GGA) or overbinding (in LDA), depending on the exchange-correlation functional.
Since GGA-PBE yields much too weak binding of PTCDA to $\operatorname{Ag}(111)$, we have decided to treat the structure within LDA. Our LDA calculations are carried out within the SIESTA package, ${ }^{41-43}$ using three layers of silver to simulate the substrate. Calculations using only two layers yield very similar structural results, thus confirming that three layers are sufficient to study the adsorption structure of PTCDA on $\operatorname{Ag}(111)$. We use a double-zeta basis set with two $s$, two $p$, and one $d$ orbital for carbon as well as for oxygen, two $s$ and one $p$ orbital for hydrogen, and two $s$, one $p$, and two $d$ orbitals for silver. By using a very small contraction energy shift of $0.002 \mathrm{eV}$ in the preparation of the orbitals, ${ }^{41}$ we achieve a spatial truncation of the most extended orbitals at 3.57, 2.81, 3.45, and $4.54 \AA$ for $\mathrm{C}, \mathrm{O}, \mathrm{H}$, and $\mathrm{Ag}$, respectively. Such a large extent of the orbitals turned out to be crucial to obtain reliable, converged results. ${ }^{17,18}$ The potential and the charge density are represented by a Fourier series with a cutoff energy of $200 \mathrm{Ry}$.

\section{B. Calculated structure of PTCDA:Ag(111)}

\section{Superstructure}

The adsorption of organic molecules on noble-metal surfaces is controlled by a subtle balance between intermolecular forces and substrate-adsorbate interaction. In the particular case of PTCDA on $\mathrm{Ag}(111)$, it turns out that one monolayer [to be precise, the (102) plane] of the $\beta$-PTCDA bulk crystal fits nearly perfectly on the $\operatorname{Ag}(111)$ surface. ${ }^{44-46}$ Within the $\beta$-phase structure, bulk PTCDA contains herringbone layers of coplanar molecules with a rectangular twodimensional unit cell of $19.30 \times 12.45 \AA^{2} .45$ The $(6,1$, $-3,5)$ superstructure of the $\operatorname{Ag}(111)$ surface, on the other hand, is given by a unit cell of $18.96 \times 12.61 \AA^{2}$, of nearly rectangular shape (with a very small deviation of $1^{\circ}$ from being rectangular), ${ }^{13}$ i.e., the two unit cells differ by only about $1.5 \%$ in both directions. As a consequence of this nearly perfect match, PTCDA can be adsorbed on $\operatorname{Ag}(111)$ as a commensurate monolayer of flat-lying molecules.

The structure is shown in Fig. 1. It consists of two molecules (labeled A and B in this work) per surface unit cell in a herringbone pattern. It is important to note that the two molecules are not equivalent ${ }^{5,13}$ (contrary to the situation in bulk PTCDA). On the one hand, the orientation angle of $77^{\circ}$ between the two molecules means that only one of them can be aligned with the $\operatorname{Ag}(111)$ lattice (molecule A in our notation), while the other molecule (B) must be misaligned by about $17^{\circ}$. On the other hand, the slight deviation of the unit cell from being rectangular causes subtle differences in the distances between intermolecular hydrogen-oxygen pairs, which play an important role for the intermolecular interaction.

\section{Adsorption sites}

Both molecules are adsorbed such that their centers are located above bridge sites between two Ag surface atoms. This behavior is revealed by total-energy minimization and by experiment. ${ }^{20}$ In our geometry optimization, all atoms are allowed to relax (including the Ag atoms which, however, 


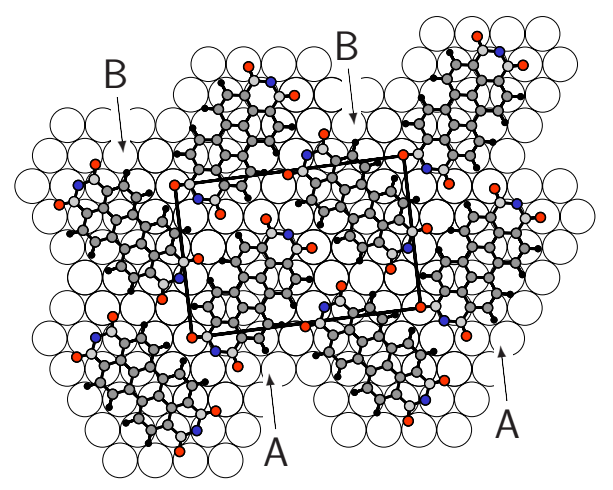

FIG. 1. (Color online) Structure of the monolayer of PTCDA on the $\operatorname{Ag}(111)$ surface. The nearly rectangular surface unit cell ( $18.96 \times 12.61 \AA^{2}$, tilted by $1^{\circ}$ ) contains two molecules. The open large circles indicate the $\mathrm{Ag}$ surface atoms. Note that one of the molecules (A) is nearly perfectly aligned with the $\operatorname{Ag}(111)$ lattice, while the other one (B) is misaligned by $17^{\circ}$. The centers of both molecules are placed on bridge sites of the $\mathrm{Ag}(111)$ substrate. For details, see text.

relax by much less than $0.05 \AA$ ), which should, in principle, result in the optimum adsorption geometry automatically. In the present case, however, fully automatic optimization is difficult since the centers of mass of the molecules hardly move laterally, although they are allowed to. This behavior results from a hierarchy of different contributions to the total energy and from the large dimension of the configuration space (228 coordinates just from the two molecules alone). The intramolecular covalent bonds are very strong and of quite simple chemical nature, leading to converged bond lengths within just a few iterations. The total-energy effects due to lifting and bending the molecule and due to vertical distortion of its end groups, on the other hand, are much smaller, thus requiring much higher accuracy in the force calculation and many more iteration steps to find the optimum. The lateral displacement of the entire monolayer finally causes extremely weak energy changes combined with very slow progress due to the vast configuration space. This means that it appears nearly impossible to relax the entire structure laterally from the forces on the atoms. Instead, we choose particular starting configurations for the lateral arrangement of the monolayer on the surface. One possible configuration is shown in Fig. 1, with the center of both molecules on bridge sites. Other starting configurations are obtained by shifting the entire monolayer laterally. In all cases, the centers of mass of the two molecules do not move noticeably even after 100 iterations, thus showing the necessity for our procedure. Table I summarizes the various starting configurations that we have tried and the resulting adsorption energies.

A final comparison between all relevant configurations clearly shows that the configuration displayed in Fig. 1 has minimum energy, with a binding energy of $5.99 \mathrm{eV}$ per two molecules. The entire procedure and especially the resulting binding energies should be taken with some care due to the problems of employing DFT-LDA in the first place (see the discussion above), in particular, due to the incorrect description of the van der Waals interaction by the DFT functional used here. The binding energy of $3 \mathrm{eV}$ per molecule is probably too large, resulting from the typical overbinding of LDA. GGA, in fact, yields much smaller binding energies of about $0.5 \mathrm{eV}$ [within PW91 (Ref. 23)] and less than $0.1 \mathrm{eV}$ (within PBE). ${ }^{17,18}$ The true binding energy of PTCDA on $\operatorname{Ag}(111)$ should, thus, be expected somewhere between the LDA and GGA results, i.e., in the range of $0.5-3 \mathrm{eV}$ per molecule. To our knowledge, there is no experimental information about the binding energy apart from the observation that PTCDA does not desorb from $\operatorname{Ag}(111)$, which indicates a binding energy of much more than $k_{B} T$ at $T>550 \mathrm{~K} .{ }^{13}$ Note that the variance of the total adsorption energy due to

TABLE I. Adsorption energies (LDA) of eight different lateral arrangements of the PTCDA monolayer on the $\operatorname{Ag}(111)$ surface, obtained from optimization starting with the configuration denoted in column 1 (see text). The first column denotes the shift of the entire monolayer relative to the optimal bridge-bridge adsorption geometry (shown in Fig. 1). The shift is expressed as a two-dimensional vector of Cartesian coordinates, given in units of the $\operatorname{Ag}(111)$ surface lattice constant $a_{0}=2.892 \AA$. The angles given in columns 3 and 5 denote the orientation of the respective molecule relative to a high-symmetry direction of the $\mathrm{Ag}(111)$ surface lattice. For all high-symmetry sites, an angle of zero means alignment of the long axis of the molecule with the direction from the site to a neighboring on-top site. The notation "nonsymmetric" indicates positions that are neither bridge, top, nor hollow.

\begin{tabular}{|c|c|c|c|c|c|}
\hline \multirow{2}{*}{$\begin{array}{l}\text { Shift with respect } \\
\text { to Fig. } 1 \\
\left(a_{0}\right)\end{array}$} & \multicolumn{2}{|c|}{ Molecule A } & \multicolumn{2}{|c|}{ Molecule B } & \multirow{2}{*}{$\begin{array}{l}\text { Binding } \\
\text { (eV/cell) }\end{array}$} \\
\hline & Site & Angle & Site & Angle & \\
\hline & Bridge & $0^{\circ}$ & Bridge & $17^{\circ}$ & -5.99 \\
\hline$(-1 / 4,-\sqrt{3} / 4)$ & Top & $0^{\circ}$ & Bridge & $-43^{\circ}$ & -5.60 \\
\hline$(1 / 4,-\sqrt{3} / 4)$ & Bridge & $60^{\circ}$ & Top & $17^{\circ}$ & -4.92 \\
\hline$(1 / 2,0)$ & Bridge & $-60^{\circ}$ & Bridge & $77^{\circ}$ & -4.94 \\
\hline$(1 / 4,-\sqrt{3} / 12)$ & Hollow & $30^{\circ}$ & Nonsymmetric & & -5.31 \\
\hline$(-1 / 4, \sqrt{3} / 12)$ & Hollow & $30^{\circ}$ & Nonsymmetric & & -5.39 \\
\hline$(1 / 4, \sqrt{3} / 12)$ & Nonsymmetric & & Hollow & $17^{\circ}$ & -5.69 \\
\hline$(-1 / 4,-\sqrt{3} / 12)$ & Nonsymmetric & & Hollow & $17^{\circ}$ & -5.74 \\
\hline
\end{tabular}


lateral displacements of the molecules is quite weak, indicating that a single PTCDA molecule could easily diffuse on the surface at sufficiently high temperature.

The lateral position should be more or less unaffected by the fundamental problems of DFT since the van der Waals interaction is expected to be relatively site insensitive. In particular, we believe that the adsorption on bridge sites is a reliable result of our calculations. It also results as the optimum adsorption geometry of DFT-PBE calculations (although the binding energies and their differences are so small that the analysis becomes hindered by numerical noise). Double-bridge-site adsorption has been confirmed by STM experiments, which allowed to identify both adsorption sites (A and B) as bridge sites to within $0.2 \AA .^{20}$

\section{Electronic structure}

We now turn to the electronic structure of the adsorbate, which is influenced by its bonding both to the substrate and to neighboring molecules.

For a detailed understanding of the substrate-bonding mechanism, it is most illustrative to consider the local density of states (LDOS) above and below the molecules. This is shown by the dashed-dotted line in Fig. 2(a) at a height of $4 \AA$ above the surface, i.e., $1.2 \AA$ above the molecules (without lateral resolution, i.e., just averaging over the surface unit cell). The LDOS is dominated by a peak at an energy of $-1.4 \mathrm{eV}$, a broad feature just below the Fermi level (at $0 \mathrm{eV}$ ), and a feature at an energy of $1 \mathrm{eV}$. By projecting the corresponding wave functions onto the molecular states of a single gas-phase molecule of PTCDA, we clearly identify these structures as originating from the highest occupied molecular orbital (HOMO), LUMO, and LUMO+1/LUMO+2 states of the free molecule. Note that the energies of the LUMO +1 and LUMO+2 states differ by less than $0.05 \mathrm{eV}$ in the gas phase, which makes it very difficult to establish their order under the influence of intermolecular interaction and adsorbate-substrate interaction. These four molecular states, which play a major role in the interpretation of the scanning tunneling images further below, are shown in Fig. 3.

At the height of $4 \AA$ above the $\operatorname{Ag}(111)$ surface, the contribution of the substrate to the local density of states (DOS) is already very weak. The influence of the substrate becomes visible in the data shown by the solid line in Fig. 2, which denotes the LDOS at a height of $1.4 \AA$ above the substrate, i.e., in the middle, between substrate and adsorbate. For comparison, the local DOS of the bare $\operatorname{Ag}(111)$ surface at the same height is shown by the dashed line. Apparently, the presence of the adsorbate enhances the bare surface LDOS at some energies (caused by the molecular states) and reduces it at other energies. We come back to these issues when discussing the substrate bonding in conjunction with Fig. 5.

The LDOS calculated with LDA [Figs. 2(a) and 2(b)] can be compared to experimental photoelectron spectroscopy and scanning tunneling spectroscopy data. Photoelectron spectroscopy shows an electronic state at $-1.6 \mathrm{eV}$ binding energy and a strongly enhanced density of states slightly below the Fermi level. ${ }^{21}$ Similarly, the STS spectra ${ }^{20}$ shown in Fig. 2(c) are in good qualitative agreement with the calculated LDOS

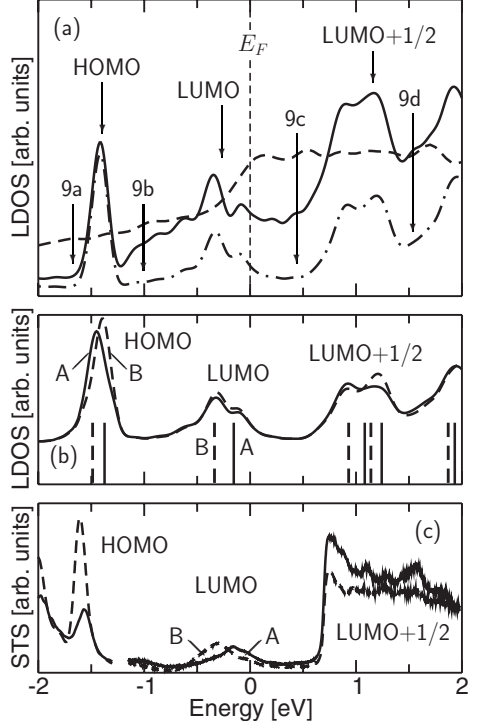

FIG. 2. (a) Laterally averaged local density of states (LDOS) of PTCDA-Ag(111), calculated $1.2 \AA$ above the PTCDA molecules (dashed-dotted line) and between the molecule and the top silver layer (1.4 $\AA$ above the Ag atom nuclei) (solid line). The dashed line indicates the LDOS of the bare $\operatorname{Ag}(111)$ surface at the same height (1.4 ̊). By wave-function projection techniques, several molecular states can be identified at energies of $-1.4 \mathrm{eV}$ (HOMO), below and around $0 \mathrm{eV}$ (LUMO), and at $1 \mathrm{eV}(\mathrm{LUMO}+1$ and LUMO+2; cf. Fig. 3). The vertical dashed line indicates the Fermi level. The labels 9a-9d refer to the discussion of Fig. 9. (b) LDOS of PTCDA$\mathrm{Ag}(111)$ above the adsorbate [cf. dashed-dotted line of (a)], evaluated separately for molecules A (solid line) and B (dashed line). A vertical offset has been used for better visibility. The vertical bars indicate the energy levels in a freestanding monolayer. Their energy scale has been shifted such that the HOMO states match the HOMO-derived structure in the adsorption system. (c) Measured tunneling spectrum above molecules A (solid line) and B (dashed line). Note that data below $U=-1.2 \mathrm{eV}$ have been obtained with a functionalized tunneling tip, while data above $U=-1.2 \mathrm{eV}$ have been measured using a bare tip (see the discussion in Sec. IV A).

spectra in Figs. 2(a) and 2(b), as far as position and width of the various features are concerned.

With respect to the STS data, two issues should, however, be noted: Firstly, in Fig. 2(c), we have combined two spectra which have been recorded with different tips. Experimentally, we find that successful spectroscopy of the HOMO requires a different tip preparation than required for the LUMO and LUMO+1/LUMO+2. As we will discuss in Sec. III, this difference can be understood on the basis of our STM simulation. Secondly, we note that while the spectral shapes of the HOMO and the LUMO in experiment and theory agree closely, there is some deviation between the calculated and measured line shapes of the LUMO $+1 /$ LUMO+2. The steplike shape of the LUMO $+1 /$ LUMO +2 in experimental STS can be traced back to the mixing of this molecular orbital with the depopulated $\mathrm{Ag}$ (111) surface state, leading to the formation of a twodimensional, nearly free delocalized interface state which exhibits the characteristic box-shaped LDOS observed in Fig. 2(c). ${ }^{22}$ Because this state is expected to penetrate quite far 


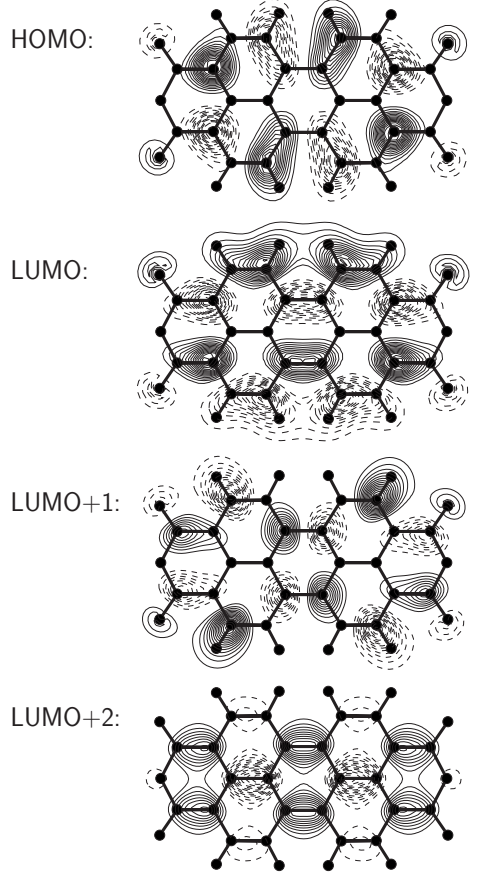

FIG. 3. Charge density of the HOMO, LUMO, LUMO+1, and LUMO +2 states of a flat PTCDA molecule in the gas phase. Note that the LUMO+1 and LUMO+2 states have nearly the same energy (to within $0.05 \mathrm{eV}$ ). The drawing plane is located $1 \AA$ above the molecule. Dashed lines indicate negative values of the wave function.

into the silver crystal [similar to the bare $\operatorname{Ag}(111)$ Shockley state], the calculation, which employs three atomic layers of $\mathrm{Ag}$, may have difficulties in predicting the correct dispersion of this interface state, which is most likely caused by wavefunction overlap in the metal rather than between the $\mathrm{LUMO}+1 / \mathrm{LUMO}+2$ orbitals in the molecular layer.

These details notwithstanding, the LDA spectrum of Fig. 2(a) is in reasonable agreement with the available experimental data. Quasiparticle corrections beyond LDA, as discussed by Dori et al. ${ }^{47}$ are relatively small, and LDA yields reasonably reliable band-structure levels of the adsorbate relative to the Fermi level of the substrate. The reason is that, on the one hand, quasiparticle corrections as a whole are less pronounced here due to metallic screening, which reduces the variance of the electron self-energies for different states. On the other hand, the LUMO level is pinned close to the Fermi level by its partial occupation. A comparison with PTCDA gas-phase spectroscopic data, both on the singleparticle level and on the two-particle level, may not be meaningful for these two reasons.

We further note that within GGA-PBE, we find spectral features that are nearly identical to the ones shown in Fig. 2, with energy differences of less than $0.1 \mathrm{eV}$. Apparently, the pinning of the LUMO to the Fermi level and the metallic screening are more important for the spectrum than the choice of the DFT functional. Of course, this only holds if the same geometry is used (e.g., LDA equilibrium geometry). At the GGA equilibrium geometry, which is characterized by much farther substrate-adsorbate distance, the mo-
TABLE II. Distances (in $\AA$ ) between carboxylic oxygen atoms on one molecule and hydrogen atoms on neighboring molecules.

\begin{tabular}{lrr}
\hline \hline Atoms & Monolayer on $\mathrm{Ag}(111)$ & $\beta$-PTCDA \\
\hline $\mathrm{O}(\mathrm{A})-\mathrm{H}(\mathrm{A})$ & 2.31 & 2.18 \\
$\mathrm{O}(\mathrm{A})-\mathrm{H}(\mathrm{B})$ & $2.05,2.19$ & $2.03,2.25$ \\
$\mathrm{O}(\mathrm{B})-\mathrm{H}(\mathrm{A})$ & $1.99,2.03$ & $2.03,2.25$ \\
$\mathrm{O}(\mathrm{B})-\mathrm{H}(\mathrm{B})$ & 2.59 & 2.18 \\
\hline \hline
\end{tabular}

lecular states would observe much different interaction with substrate states and the spectrum would be much different.

One of the key features of the adsorption of PTCDA on $\mathrm{Ag}(111)$ is the fact that the molecular LUMO state becomes partially occupied. ${ }^{8,11,14,20,21}$ This is clearly visible in the LDOS, which shows the LUMO state as a broad feature around and below the Fermi level. A closer analysis of the corresponding redistribution of electronic charge reveals that a substantial charge transfer from the substrate to the molecules of about 0.35 electrons per molecule occurs (see the discussion of Fig. 5 below). This partial occupation of the previously unoccupied LUMO state and the resulting charge transfer are of prime importance both for the structural properties and for the STM images of the system. In particular, this mechanism constitutes a substantial part of the binding to the substrate. ${ }^{15}$

Since the two molecules in the unit cell are structurally inequivalent, one may ask to which extent this translates into different electronic structures of the A and B molecules. Two possible influences must be distinguished: Firstly, the different orientations of the two molecules with respect to the substrate may modify the electronic structure; e.g., for the perfectly aligned molecule $\mathrm{A}$, the carboxylic oxygen atoms (at the corner of each molecule) are situated directly above $\mathrm{Ag}$ atoms, while for the misaligned molecule B, the carboxylic oxygen atoms are in significantly displaced lateral positions, i.e., not on top of Ag atoms. Since it turns out that the interaction of the carboxylic oxygen atoms with the $\mathrm{Ag}$ atoms underneath contributes significantly to the adsorption process (see below), this structural difference between $\mathrm{A}$ and B molecules may well result in distinct electronic structures. Secondly, different intermolecular hydrogen-oxygen bond lengths may lead to a type-specific electronic structure. Several such bonds with bond lengths of slightly more than $2 \AA$ occur (see Fig. 1), and Table II lists the various calculated hydrogen-oxygen distances. The table, indeed, reveals a substantial asymmetry between molecules A and B. In particular, the bonds between $\mathrm{O}$ atoms of molecule $\mathrm{B}$ and $\mathrm{H}$ atoms of molecule $\mathrm{A}[\mathrm{O}(\mathrm{B})-\mathrm{H}(\mathrm{A})]$ are significantly shorter than the bonds between $\mathrm{O}$ atoms of molecule $\mathrm{A}$ and $\mathrm{H}$ atoms of molecule $\mathrm{B}[\mathrm{O}(\mathrm{A})-\mathrm{H}(\mathrm{B})]$ (see Table II). The main driving force for this effect is the $1^{\circ}$ distortion of the unit cell from being rectangular, which distorts the arrangement of the two molecules relative to each other.

The asymmetry of intermolecular $\mathrm{O}-\mathrm{H}$ bonds contributes to the nonequivalent electronic levels of molecules $\mathrm{A}$ and B. ${ }^{20}$ To illustrate this, the vertical lines in Fig. 2(b) show the LDOS of a freestanding monolayer of PTCDA in the configuration as found in the adsorption system. Different from a 
symmetric arrangement in a rectangular unit cell, the electronic levels on the two molecules are shifted relative to each other by about $0.1-0.2 \mathrm{eV}$, with the levels of molecule $\mathrm{A}$ being higher in energy than those of molecule B. Since both molecules have nearly the same internal structure, this effect is exclusively caused by the different $\mathrm{H}$ bonds.

Interestingly enough, the order of the levels is changed again when the interaction with the substrate is additionally taken into account. ${ }^{20}$ To show this, the upper curves in Fig. 2(b) display the local DOS of the two molecules in the adsorption system [same data as given by the dashed-dotted line in Fig. 2(a), but separately for each molecule]. In the case of the HOMO state, the order of HOMO-A and HOMO-B is changed. Apparently, the different alignment of the two molecules with the substrate leads to spectral shifts stronger than those induced by the $\mathrm{H}$ bonds, such that HOMO-A is finally found $0.05 \mathrm{eV}$ below HOMO-B. In the case of the LUMO and LUMO+1/LUMO+2 states, which show more complex hybridization with Ag substrate states, the structure of their spectral features is slightly different for the two molecules. Again, this is caused by the different alignments of the two molecules with the substrate. These effects visible in the LDOS data are of importance for the STM images as discussed further below.

One should note that the type-specific electronic structure is extremely subtle and may well constitute the limit of the reliability of the calculations. They may depend on the specifications of the DFT calculation (code, pseudopotential, basis set, and exchange-correlation functional) and may be subject to many-body effects beyond the present theory. Therefore, these slight differences between the spectra of molecules A and B should be taken with care. Most importantly, however, our results clearly indicate that both the distortion of the surface unit cell and the different alignments of the molecules with the substrate lattice give rise to such spectral differences, which have, indeed, been found in STM experiments, ${ }^{20}$ as Fig. 2(c) shows. Note, however, that different from the calculation, the experiment has the HOMO of molecule B below the HOMO of molecule A. This just indicates that the relative magnitudes of the intermolecular and interfacial effects come out differently in the calculation and in the experiment.

Evidently, intermolecular bonds are also crucially important for understanding the differences between the adsorption of a full monolayer and the adsorption of a single PTCDA molecule, which is unaffected by such hydrogen-oxygen bonds.

\section{Bonding mechanism}

Qualitatively, the intramolecular structure of PTCDA remains unchanged during adsorption. For a weakly chemisorbed molecule this is indeed expected, because the internal covalent bonds of the molecule are much stronger than the bond with the substrate. However, several subtle, characteristic changes do take place, and it turns out that these are very revealing with regard to the bonding mechanism of PTCDA on $\operatorname{Ag}(111)$. To illustrate this, we discuss in Table III the intramolecular bond lengths for a gas-phase molecule, a
TABLE III. Intramolecular bond lengths (in $\AA$ ) of PTCDA in the gas phase, of a single PTCDA molecule adsorbed on $\operatorname{Ag}(111)$ with no contact with other molecules, and of PTCDA molecules adsorbed as a monolayer on $\mathrm{Ag}(111)$. All calculations were performed within DFT-LDA. For the labeling of the bonds, we refer to Fig. 4. In the case of the monolayer, the bond length has been averaged over the two molecules. The data in column 3 have been obtained by Dori et al. (Ref. 47) within DFT-LDA for the free molecule in the gas phase.

\begin{tabular}{lcccc}
\hline \hline & \multicolumn{2}{c}{ Gas phase } & & \\
\cline { 2 - 3 } Bond & This work & Ref. 33 & $\begin{array}{c}\text { Single } \\
\text { adsorbed }\end{array}$ & Monolayer \\
\hline C-C (1) & 1.463 & 1.45 & 1.441 & 1.439 \\
C-C (2) & 1.405 & 1.39 & 1.429 & 1.436 \\
C-C (3) & 1.431 & 1.42 & 1.437 & 1.440 \\
C-C (4) & 1.400 & 1.39 & 1.392 & 1.393 \\
C-C (5) & 1.427 & 1.42 & 1.438 & 1.439 \\
C-C (6) & 1.392 & 1.38 & 1.418 & 1.426 \\
C-C (7) & 1.414 & 1.40 & 1.421 & 1.426 \\
C-C (8) & 1.475 & 1.46 & 1.443 & 1.439 \\
C-O (9) & 1.214 & 1.19 & 1.239 & 1.244 \\
C-O (10) & 1.384 & 1.37 & 1.387 & 1.388 \\
\hline \hline
\end{tabular}

single adsorbed molecule, and a full monolayer. The labeling of the bonds is shown in Fig. 4.

One of the most interesting features of the bond-length data presented in Table III is the apparent interrelation with the electronic structure upon adsorption. Gas-phase PTCDA shows C-C bond lengths between 1.39 and $1.48 \AA$, indicating varying degrees of $\pi$ conjugation and double-bond char-

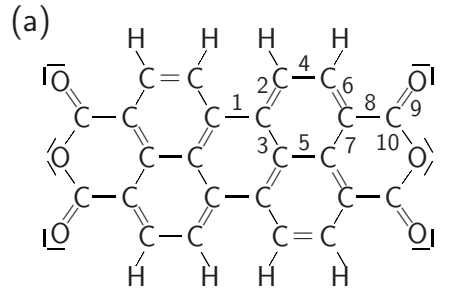

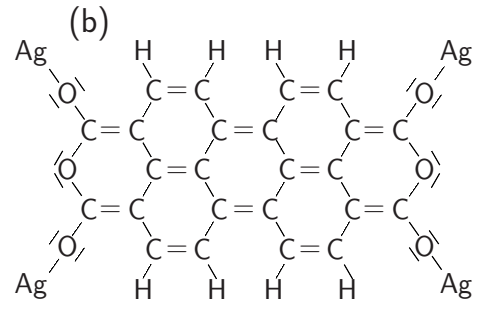

FIG. 4. Structure of PTCDA in two extreme situations. (a) refers to the gas-phase molecule, while (b) refers to the adsorbed molecule in the hypothetical limit of forming full covalent bonds between the carboxylic oxygen atoms and the silver surface, causing drastic changes in the single and double bonds and breaking the conjugation structure of the perylene backbone. The labels refer to the bond lengths as given in Table III. 
acter. As noted before, a main driving force of the adsorption of PTCDA on $\operatorname{Ag}(111)$ is the partial occupation of the former LUMO state. Since the LUMO has maxima at the $\mathrm{C}-\mathrm{C}$ bonds 1,4 , and 8 , the double-bond character of these bonds is enhanced and the bond length is shortened by this mechanism. On the other hand, the LUMO is of antibonding character for bonds 2 and 6 (where the LUMO has nodes), which are, thus, elongated due to occupation of the LUMO (decreasing bond order). These effects are, indeed, found in the data of Table III.

Remarkably, the same subtle changes of the C-C bond lengths can also be explained by the formation of weak covalent bonds between the carboxylic oxygen atoms and the $\mathrm{Ag}$ substrate underneath. This is demonstrated in Fig. 4. Simple bond counting shows that the formation of full $\sigma$ bonds between $\mathrm{Ag}$ and $\mathrm{O}$, one at each of the four corners of the molecule, would turn the $\mathrm{C}-\mathrm{O}$ double bonds of the carboxylic oxygen atoms (labeled "9") into single bonds, which would then change the C-C bond characters of the entire molecule (see Fig. 4), breaking the $\pi$ conjugation and causing the same type of bond-length changes as obtained from the occupation of the LUMO. Apparently, both mechanisms lead to the same geometrical changes within the PTCDA molecule and, thus, support each other rather than compete with one another. This explains the strong and efficient bonding of PTCDA to $\operatorname{Ag}(111)$. The formation of weak $\mathrm{Ag}-\mathrm{O}$ bonds and corresponding slight reduction of the $\mathrm{C}=\mathrm{O}$ bond toward a single bond are also reflected in the stretching of bond 9 by $0.03 \AA$ upon adsorption. It should be noted here that vibrational spectroscopy reveals that the $\mathrm{C}=\mathrm{O}$ bond stretching frequency is substantially softened. ${ }^{8}$

Further information about the binding of the molecule to the substrate is obtained by charge difference plots in which the electronic charge of the adsorption system is compared with the sum of the charge densities of its two noninteracting components. ${ }^{15,18}$ Figure 5(a) displays a cross section of this quantity, integrated along the direction given by the short axis of the molecule. For further illustration, Fig. 5(b) shows the total charge density as a line plot, i.e., $\rho(z)=\int \rho(\mathbf{r}) d x d y$, integrated laterally over the surface unit cell. Note that the integration of this line density along $z$ correctly yields a total of 280 electrons for the two PTCDA molecules (at $z$ $=2.8 \AA$ ) and 363 electrons for each $\mathrm{Ag}(111)$ layer (consisting of $33 \mathrm{Ag}$ atoms per layer in the surface unit cell). Apparently, the total charge density is mostly given by the sum of the charge densities of $\operatorname{Ag}(111)$ alone and the molecular layer alone, indicated by the dashed curves in Fig. 5(b). The effect of the substrate-adsorbate interaction becomes visible in Fig. $5(\mathrm{c})$, which shows again the difference, i.e., $\Delta \rho(\mathbf{r})$ $:=\rho_{\text {PTCDA:Ag(111) }}(\mathbf{r})-\rho_{\text {PTCDA }}(\mathbf{r})-\rho_{\operatorname{Ag}(111)}(\mathbf{r}) \quad$ [cf. Fig. 5(a)], but now as a line density after lateral integration. The data shown in Fig. 5(c), thus, correspond to those in Fig. 5(a).

Apparently, the main effect of the charge redistribution is a reduction of electronic charge density directly above the $\operatorname{Ag}(111)$ substrate top layer ( $1 \AA$ above the $\mathrm{Ag}$ nuclei) and an increase inside the molecule. This increase occurs about $1 \AA$ above and below the atomic nuclei, i.e., mostly in the carbon and oxygen $p_{z}$ orbitals, accompanied by a slight decrease in the plane of the nuclei [at $z=2.8 \AA$ in Fig. 5(c)]. This charge

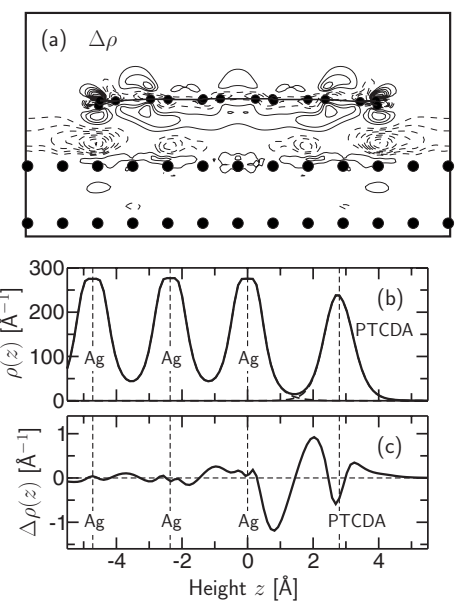

FIG. 5. (a) Side view at the charge density difference of a single PTCDA molecule adsorbed at the $\operatorname{Ag}(111)$ surface. The quantity displayed in (a) is given by $\Delta \rho(\mathbf{r}):=\rho_{\text {PTCDA: } \operatorname{Ag}(111)}(\mathbf{r})-\rho_{\text {PTCDA }}(\mathbf{r})$ $-\rho_{\mathrm{Ag}(111)}(\mathbf{r})$, i.e., the difference between the self-consistent LDA charge density of the adsorption system minus the charge densities of the individual components [PTCDA molecule, $\operatorname{Ag}(111)$ surface alone]. For visualization, $\Delta \rho$ has been integrated along the axis perpendicular to the drawing plane. Solid (dashed) lines correspond to positive (negative) electronic charge transfer, indicated by level spacing of 0.0044 particles per $a_{B}^{2}$. (b) Total charge density $\rho(z)$ obtained by lateral integration over the surface unit cell. The solid line refers to the adsorption system, while the two dashed lines (hardly visible) refer to the $\mathrm{Ag}(111)$ surface alone and the PTCDA moleculal layer alone, respectively. (c) Difference charge density $\Delta \rho(z)$ given by the difference of the three curves shown in (b) [i.e., PTCDA:Ag(111) minus $\mathrm{Ag}(111)$ minus PTCDA]. This quantity corresponds to the data shown in (a).

increase is significantly asymmetric, i.e., it mostly occurs below the molecule. To a large degree, it is given by the partial occupation of the formerly empty LUMO state; however, the LUMO state is apparently influenced and modified by the substrate, since the LUMO on its own would constitute the same charge increase below and above the molecule. Obviously, the state in question results from hybridization of the LUMO and of $\operatorname{Ag}(111)$ states extending into vacuum. For a quantitative evaluation of the charge transfer, we take the node of $\Delta \rho(z)$ near $z_{0}=1.4 \AA$ (which happens to be halfway between substrate and molecule) as a reference point [see Fig. 5(c)]. The charge reduction below $z_{0}$ (i.e., in the substrate) as well as the charge enhancement above $z_{0}$ (i.e., in the molecule) both amount to 0.35 electrons (per molecule), which constitutes our estimate of the net charge transfer from the substrate to the adsorbate.

Note that the nearly complete occupation of the LUMO (cf. Fig. 2) would mean a charge transfer of nearly two electrons. As a consequence of the complex hybridization processes between the substrate and molecular states, the charge transfer as defined here is significantly smaller.

Particularly strong changes of the electronic charge density are found at and underneath the carboxylic oxygen atoms [see Fig. 5(a)], clearly indicating the formation of local bonding between oxygen and silver atoms.

It should be noted that the charge transfer depends on the approximations of the theoretical framework. For instance, 
TABLE IV. Height of atoms above the $\operatorname{Ag}(111)$ surface (in $\AA$ ), resulting from DFT-LDA. All carbon atoms have approximately the same height, while significant splitting between the anhydride and carboxylic oxygen atoms is observed.

\begin{tabular}{|c|c|c|c|c|}
\hline & \multirow{2}{*}{$\begin{array}{c}\text { Single } \\
\text { molecule }\end{array}$} & \multicolumn{2}{|c|}{ Monolayer } & \multirow{2}{*}{$\begin{array}{c}\text { Expt. } \\
\text { (Ref. 15) }\end{array}$} \\
\hline & & A & $\mathrm{B}$ & \\
\hline Carbon, average & 2.76 & 2.70 & 2.64 & 2.86 \\
\hline Carbon, max-min & 0.15 & 0.14 & 0.07 & \\
\hline Anhydride $\mathrm{O}$ & 2.68 & 2.78 & 2.72 & 2.97 \\
\hline Carboxylic $\mathrm{O}, 1 \mathrm{H}$ bond & & 2.47 & 2.57 & \\
\hline Carboxylic $\mathrm{O}, 2 \mathrm{H}$ bond & & 2.55 & 2.52 & \\
\hline Carboxylic $\mathrm{O}$, average & 2.44 & 2.51 & 2.55 & 2.68 \\
\hline Anhydride O-carbon & 0.08 & 0.08 & 0.08 & 0.11 \\
\hline Carboxylic O-carbon & -0.32 & -0.19 & -0.13 & -0.18 \\
\hline
\end{tabular}

DFT calculations employing the Perdew-Wang GGA functional yield no significant charge transfer, ${ }^{23}$ demonstrating that such quantities are very sensitive to the approach and may not be fully described by any DFT calculation.

\section{Adsorption height and vertical distortion}

The adsorption height of the molecule above the substrate and the molecular distortion are closely related to the substrate-bonding mechanism. Experimentally, a close correlation between bonding strength and adsorption height has been established, ${ }^{24,48}$ and it has also been found that the molecular distortion of PTCDA varies significantly with substrate reactivity. ${ }^{24,48,49}$ The height of the molecule as a whole is difficult to address within DFT due to the abovementioned problems of the exchange-correlation functional in describing the van der Waals interaction. Nonetheless, it is very instructive to discuss the resulting vertical positions. The total height of the molecule may be expressed by the average height of its carbon atoms. Here, we obtain a value of $2.76 \AA$ for a single molecule and $2.70 \AA$ (2.64 $\AA$ ) for a molecule of type A (B) within the monolayer (see Table IV). This compares reasonably well with the experimental data of $2.86 \AA$ for the monolayer system, as obtained from NIXSW measurements. ${ }^{15}$ The remaining differences of about 0.1-0.2 $\AA$ are due to the overbinding problems of DFT-LDA for weakly interacting systems, in which van der Waals interaction plays a major role.

It is also interesting to note in the present context that NIXSW experiments on a disordered metastable lowtemperature phase of PTCDA on $\mathrm{Ag}(111)$, in which the molecules can, to a first approximation, be regarded as separated, reveal a metal-molecule bond length and a molecular distortion different from the ordered monolayer. ${ }^{50}$ In agreement with the theoretical prediction, the distortion of the separate molecule is larger than that of the monolayer molecule. However, in experiment, the overall bonding distance of the separate molecule is smaller than that of the monolayer molecule.

More reliable than the total height is the height of individual atoms relative to each other, reflecting distortion of the molecule from its original flat geometry. In particular, the carboxylic oxygen atoms are significantly attracted to the surface (see Table IV), indicating substantial binding to the silver atoms underneath. Note that the carboxylic oxygen atoms do not all have the same height. On the one hand, they are involved in different numbers of hydrogen bridge bonds. For molecule $\mathrm{A}$, the $\mathrm{O}$ atom which is involved in two such bonds seems to have less possibility to interact with its $\mathrm{Ag}$ neighbor, resulting in a larger Ag-O bond length. On the other hand, the oxygen atoms of molecule B are not located directly on top of $\mathrm{Ag}$ atoms, which seems to reduce the $\mathrm{Ag}-\mathrm{O}$ interaction and to cause larger perpendicular Ag-O bond length than for molecule A $(2.55 \AA$ on average as compared to $2.51 \AA$ for molecule A). Different from the carboxylic O atoms at the corners of the molecule, the anhydride $\mathrm{O}$ atoms are repelled from the substrate rather than attracted, i.e., they do not contribute to local binding.

It is gratifying to see that the relative height of all oxygen atoms relative to the carbon atoms agrees well with the experimental data, ${ }^{15}$ thus confirming that the present DFT-LDA approach is sufficiently accurate to discuss relative positions. The molecular distortion can also be discussed as a very tangible consequence of the existence of the two interaction channels of PTCDA with the $\operatorname{Ag}(111)$ substrate (carboxylic oxygen atoms vs $\pi$ electrons). Based on experimental data of PTCDA on various surfaces with different reactivities, ${ }^{24,48,49}$ it seems that the height of oxygen is more or less the same above all surfaces (within $0.1 \AA$ ), while the height of the perylene backbone varies from surface to surface. Since the oxygen bonds can easily be bent relative to the rest of the molecule, individual height adjustment of the oxygen atoms and of the backbone immediately yields molecular distortion varying from surface to surface. ${ }^{24,48,49}$

There have been several other theoretical studies concerning the adsorption of PTCDA on $\mathrm{Ag}(111)$, mostly within density-functional theory as well. These calculations share some common trends, but differ in a number of details. In particular, the resulting adsorption height of the molecule above the substrate, the internal distortion of the molecule, and the adsorption energy vary over a large range. Picozzi et al. ${ }^{10}$ by using DMOL package, found no binding within PBE but binding at a distance of $2.8 \AA$ within LDA, accompanied by a transfer of electronic charge from the substrate to the molecule. These results indicate that occupation of the LUMO is essential. Du et al., on the other hand, by using VASP and within PW91, have found no significant charge transfer and concluded instead that bonding occurs primarily via $\mathrm{O}-\mathrm{Ag}$ bonds at the corners of the molecule. ${ }^{23}$ In a previous study within PBE-DFT, employing the SIESTA package, we concluded that both mechanisms are present, leading to significant charging and bending. ${ }^{15,17,18}$

\section{COMPUTATIONAL APPROACH TO SCANNING TUNNELING MICROSCOPY}

We now turn our attention to the corresponding scanning tunneling features (STM and STS).

The scanning tunneling data are obtained within the framework of the Tersoff-Hamann theory, ${ }^{35}$ i.e., by evaluat- 
ing the wave functions of the sample at the position of the STM tip. This approach is based on the assumption that a single electronic orbital of the tip, which is significantly broadened in energy, carries the entire tunneling current. While this assumption is certainly a crude approximation, it often appears justified since in many experiments knowledge about the details of the tip is limited, not allowing for a more detailed modeling anyway. Within the Tersoff-Hamann theory, the tunneling current (for bias voltage $U$ ) is simply given by an energy-integrated LDOS at the tip position $\mathbf{r}$ (Ref. 35):

$$
I(U) \sim \sum_{n \mathbf{k}}\left|\hat{\mathcal{O}} \psi_{n \mathbf{k}}(\mathbf{r})\right|^{2} \Theta\left(E_{n \mathbf{k}} \text { between } E_{F} \text { and } E_{F}+U\right) .
$$

The function $\Theta$ indicates that the summation is restricted to states with energy $E_{n \mathbf{k}}$ between the Fermi energy $E_{F}$ and $E_{F}+U$. For negative (positive) voltage, only occupied (empty) sample states contribute. The position $\mathbf{r}$ denotes the position at which the tip orbital is centered. The operator $\hat{\mathcal{O}}$ refers to the spatial properties of the tip orbital responsible for the tunneling process. If this tip state is of $s$-like character, the operator is unity, i.e., one just has to evaluate the sample wave functions at the position of the tip apex. If the tip state possesses higher angular momentum (e.g., $p_{z}$ or $d_{3 z^{2}-r^{2}}$, the tunneling matrix element between the tip and the surface states involves spatial derivatives of $\psi(\mathbf{r}){ }^{36,38}$ In the $p(d)$ shell, the $p_{z}\left(d_{3 z^{2}-r^{2}}\right)$ tip orbital is most relevant since they have the farthest spatial extent toward the sample. ${ }^{36} \mathrm{~A}$ $d_{3 z^{2}-r^{2}}$ orbital should be of particular relevance if the outermost tip atom is a transition-metal element. Depending on the tip orbital $\left(s, p_{z}\right.$, or $\left.d_{3 z^{2}-r^{2}}\right)$, the operator in Eq. (1) is chosen as

$$
\hat{\mathcal{O}}_{s}=1, \quad \hat{\mathcal{O}}_{p_{z}}=\frac{\partial}{\partial z}, \quad \hat{\mathcal{O}}_{d_{3 z^{2}-r^{2}}}=3 \kappa^{-2} \frac{\partial^{2}}{\partial z^{2}}-1,
$$

with $\kappa=\sqrt{2 m / \hbar^{2}\left(V_{v a c}-E_{n \mathbf{k}}\right)} \cdot{ }^{36}$

The numerical evaluation of $\psi_{n \mathbf{k}}(\mathbf{r})$ at the relevant height of several angstroms above the surface poses a significant problem to DFT because the wave functions decay exponentially into the vacuum region (by 1 order of magnitude per $1-2 \AA$ as a rule of thumb). ${ }^{40}$ The numerical value of $\psi_{n \mathbf{k}}(\mathbf{r})$, thus, becomes extremely small and is very difficult to converge. Even then, the results for the wave functions might still suffer from numerical noise. Furthermore, when localized basis sets are used (as in the present case), the basis functions are not meaningful in the far vacuum region. STM simulations have, thus, often been carried out very close to the surface, which systematically falsifies the results (see Sec. IV below).

Instead, we proceed as follows in the present work. First, a reference plane (parallel to the surface) is chosen slightly above the surface, i.e., at a height $z_{0}$ at which the wave functions $\psi_{n \mathbf{k}}\left(x, y, z_{0}\right)$ have large numerical values and are accurately represented by the basis functions. Each function can be expanded in a two-dimensional Fourier series,

$$
\psi_{n \mathbf{k}}(x, y, z)=\sum_{\mathbf{G}} a_{\mathbf{G}}(z) \exp \left\{i\left[\left(k_{x}+G_{x}\right) x+\left(k_{y}+G_{y}\right) y\right]\right\},
$$

with $\mathbf{G}=\left(G_{x}, G_{y}\right)$ being two-dimensional reciprocal lattice points of the surface. For $z=z_{0}$, the coefficients $a_{\mathbf{G}}\left(z_{0}\right)$ are easily obtained as the two-dimensional Fourier transform of $\psi_{n \mathbf{k}}\left(x, y, z_{0}\right)$ in $x$ and $y$. In order to calculate $a_{\mathbf{G}}(z)$ from $a_{\mathbf{G}}\left(z_{0}\right)$, we further assume that the crystal potential (which is negative inside the substrate) has already converged to $V_{v a c}$ at $z_{0}$ and remains $V_{\text {vac }}$ further outside. ${ }^{37,38,51}$ The Schrödinger equation, which controls the spatial propagation of $\psi$, thus, simply becomes

$$
-\frac{\hbar^{2}}{2 m} \Delta_{\mathbf{r}} \psi_{n \mathbf{k}}(x, y, z)=\left(E_{n \mathbf{k}}-V_{v a c}\right) \psi_{n \mathbf{k}}(x, y, z)
$$

for $z>z_{0}$, with $E_{n \mathbf{k}}$ being the Kohn-Sham band-structure energy of the state, as given by the slab calculation. ${ }^{52}$ Quasiparticle corrections to $E_{n \mathbf{k}}$ beyond the Kohn-Sham energies, which are of great importance for many systems to obtain reliable band-structure data, are neglected in the present study (see the discussion in Sec. II B). For each Fourier component of $\psi_{n \mathbf{k}}(x, y, z)$, the Schrödinger equation (4) implies an ordinary differential equation for $a_{\mathbf{G}}(z)$ :

$$
\frac{\partial^{2}}{\partial z^{2}} a_{\mathbf{G}}(z)=\left[(\mathbf{k}+\mathbf{G})^{2}-\frac{2 m}{\hbar^{2}}\left(E_{n \mathbf{k}}-V_{v a c}\right)\right] a_{\mathbf{G}}(z),
$$

which [for $E_{n \mathbf{k}}-V_{v a c}<0$ and assuming that $V\left(z>z_{0}\right)=V_{\text {vac }}$ $=$ const $]$ has the simple exponentially decaying solution

$$
a_{\mathbf{G}}(z)=a_{\mathbf{G}}\left(z_{0}\right) \exp \left[-\lambda\left(z-z_{0}\right)\right],
$$

with

$$
\lambda=\sqrt{(\mathbf{k}+\mathbf{G})^{2}-\frac{2 m}{\hbar^{2}}\left(E_{n \mathbf{k}}-V_{v a c}\right)} .
$$

The corresponding exponentially increasing solution is not considered since it contradicts normalization. Finally, after evaluating each Fourier component $a_{\mathbf{G}}(z)$ at the desired $z$ (i.e., the STM tip height), we can easily obtain $\psi_{n \mathbf{k}}(x, y, z)$ as the two-dimensional Fourier series of Eq. (3). By the same procedure, spatial derivatives $\left(\partial^{n} / \partial z^{n}\right)$ of $\psi_{n \mathbf{k}}(x, y, z)$ are obtained (for a non- $s$-like tip).

In addition to the tunneling current and resulting topography, one can easily investigate tunneling spectroscopy, as well, by discussing the (position dependent) derivative of current versus voltage, $\partial I / \partial U$. Following Eq. (1), it is simply given by

$$
\partial I(U) / \partial U \sim \sum_{n \mathbf{k}}\left|\hat{\mathcal{O}} \psi_{n \mathbf{k}}(\mathbf{r})\right|^{2} \delta\left(E_{n \mathbf{k}}, E_{F}+U\right) .
$$

\section{CALCULATED SCANNING TUNNELING MICROSCOPY AND SCANNING TUNNELING SPECTROSCOPY DATA}

In order to visualize the electronic structure of an adsorbed molecule, scanning tunneling images are invaluable. 
In this section, we discuss the dependence of such images on various tunneling conditions and present a systematic comparison with experimental data.

Our structural data discussed in Sec. II are still in slight disagreement with experiment, particularly with respect to the height of the molecule and the distortion at the oxygen end groups. In order to obtain reliable scanning tunneling simulation data, we have chosen to correct these deficiencies. To this end, we set the height of the central carbon atoms to $2.90 \AA$ and the height of the oxygen atoms to $2.97 \AA$ (anhydride) and $2.68 \AA$ (carboxylic), respectively (cf. Table IV), followed by relaxation of the molecules under these constraints, which yields the correct average height of $2.86 \AA$ of the $\mathrm{C}$ atoms. ${ }^{15}$ Note that these modifications only affect the vertical geometry of the system, not the lateral physics which remains unchanged. In particular, the structural data as discussed in Fig. 1 and Table III are still valid. We consider the structure obtained in this way as the most realistic geometry we can attain with our tools, serving as the starting point for the calculation of scanning tunneling data.

\section{A. Scanning tunneling microscopy topographies at various tip heights}

In experiment, topographic images are usually recorded in constant-current mode at fixed voltage, taking the tip height $Z(x, y)$ as the observable. Figure 6 shows a series of such images, calculated from Eq. (1), recorded for different maximum tip heights and tip orbitals, at a negative voltage of $U$ $=-1 \mathrm{eV}$. For a more quantitative analysis, the same data are displayed in Fig. 7 for positions along the line indicated in Fig. 6(e). In principle, there is a one-to-one correspondence between the maximum tip height in Figs. 6 and 7 and the tunneling current. This relationship is, however, difficult to evaluate due to missing information about the details of the tip. For an $s$-like tip, we find in our data an exponential decay of the current with a factor of 10 per $\Delta z=0.9 \AA$ tipheight increase. For a $p_{z}$-like $\left(d_{3 z^{2}-r^{2}}\right.$-like $)$ tip, the current decreases by a factor of 10 per $\Delta z=0.8 \AA(0.75 \AA)$ tip-height increase. All these values are consistent with a simple estimate for a one-dimensional tunneling barrier near the Fermi level. This yields $I \sim \exp \left(-2 \sqrt{2 m W / \hbar^{2}} z\right)$, which decreases by a factor of 10 per $\Delta z=\ln 10 /\left(2 \sqrt{2 m W / \hbar^{2}}\right)$. In here, $W$ denotes the work function, which amounts to $4.8 \mathrm{eV}$ for $\mathrm{Ag}$, yielding $\Delta z=1.0 \AA$ A. In our experimental procedure, we can bring the tip in mechanical contact with the molecule [which we estimate to happen at a tip-molecule distance of $2.5 \AA$, i.e., about $5.5 \AA$ above the $\operatorname{Ag}(111)$ surface layer] and then systematically increase the distance. This allows one to establish a direct relationship between the tip height and the tunneling current (see Ref. 20 for details). Using those data, we roughly estimate that the images shown in Fig. 6 (recorded at $U=-1 \mathrm{eV}$ ) would correspond to tunneling currents of about $10 \mathrm{~mA}, 300 \mathrm{nA}, 10 \mathrm{nA}, 1 \mathrm{nA}$, and $0.1 \mathrm{nA}$ (see also Fig. 8). Note that this distance-current relationship might be different for other tip preparation procedures.

STM topographies of a surface depend very sensitively on the height of the tip above the sample. The basic effect of the tunneling barrier, i.e., the fast decrease of current for increas-
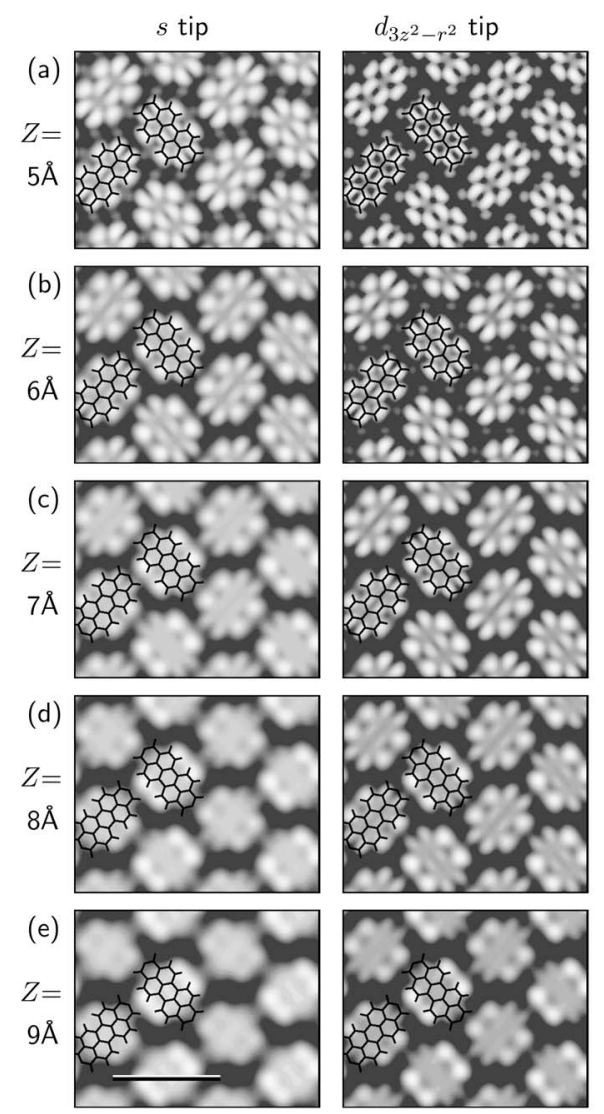

FIG. 6. Calculated constant-current topographical image of a monolayer of PTCDA on the $\operatorname{Ag}(111)$ surface, recorded with various tip orbitals and at various maximum tip heights $Z_{\text {max }}$ (ranging from 5 to $9 \AA$ above the $A g$ atoms). The maximum tip height is defined as the distance between the center of the tip orbital and the nuclei of the $\mathrm{Ag}$ surface atoms, and refers to the maximum tip height achieved for a given current. The tunneling voltage has been set to $-1 \mathrm{~V}$ in all images. (a)-(e) display constant-current images with a height difference of $1.0 \AA(0.8,0.7,0.6$, and $0.4 \AA)$ between black and white. The line in the left-hand panel for $Z=9 \AA$ indicates the line along which the data in Fig. 7 are displayed. Topography employing a $p_{z}$-like tip (not displayed here) yields images in between those of the $s$ - and $d_{3 z^{2}-r^{2}}$-like tips.

ing height, would not change the corrugation of the topography. Such changes of the corrugation arise because the wave functions are composed of different Fourier components G, as discussed in detail below. In the present case, we observe that the images change qualitatively when the tip-sample distance is increased.

As an example, we show in Fig. 6 constant-current images obtained for maximum tip heights $Z_{\max }$ in the range from 5 to $9 \AA$ (at a voltage of $-1 \mathrm{eV}$ ). At small distances, the maxima of tunneling current come from six lobes along the long central axis of each molecule, accompanied by four lobes near the hydrogen atoms. These features are easily identified as originating from the LUMO wave function (cf. Fig. 3). At $U=-1 \mathrm{eV}$, the (formerly empty) LUMO dominates the topography since it is the only molecular state with nonzero occupation between $E_{F}-1 \mathrm{eV}$ and $E_{F}$. Note that the states of the metal substrate do not contribute directly since 


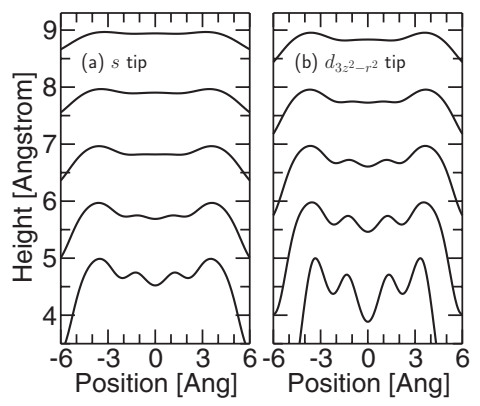

FIG. 7. Calculated constant-current profiles across molecule B

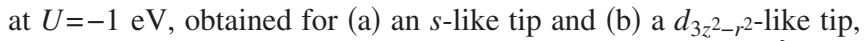
recorded for maximum tip height $Z_{\max }$ ranging from 5 to $9 \AA$. The data are the same as in Fig. 6, displayed along the line indicated in Fig. 6(e) (left panel).

they are too far away from the STM tip. At larger distances, however, the features along the central axis of the molecule die off rapidly, while the features near the hydrogen atoms merge into two elongated peanut-shaped lobes at each side of the molecule, which finally dominate the topographic image. Consequently, the spatial details of the LUMO wave function, which are clearly recognizable at small distances, wash out at larger distance and the structural details are lost. In addition to the loss of spatial resolution, the various spatial features of the molecule change their relative amplitude, i.e., the hydrogen side groups dominate the image at large tipsample distances. At first glance, one might even mistake these features as originating from the oxygen-dominated end groups of the molecule, which would result in a severe mis-
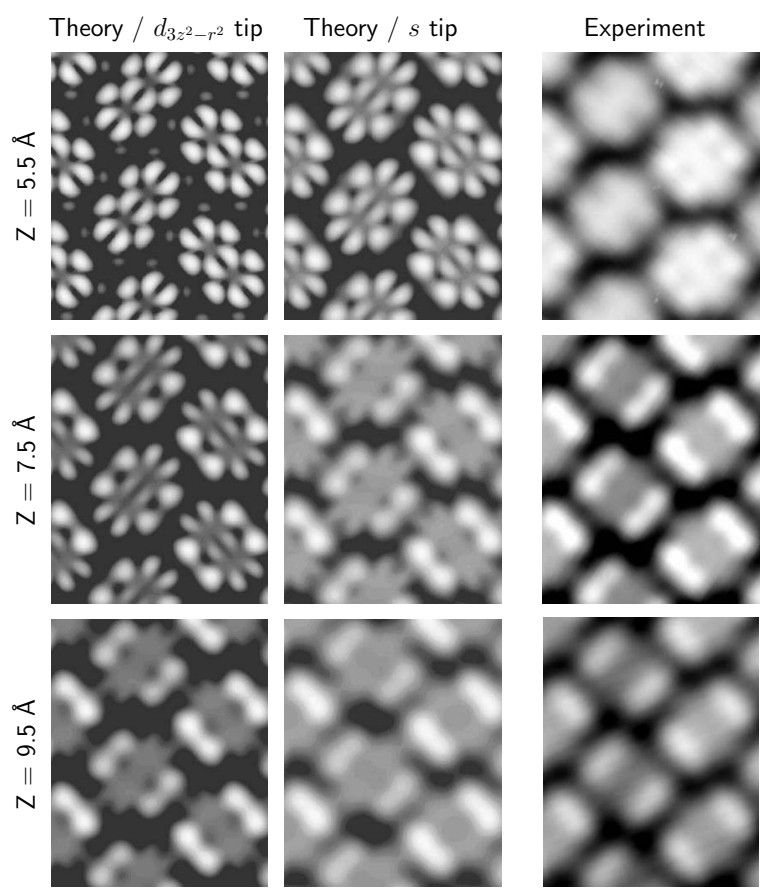

FIG. 8. Calculated and measured constant-current topographical images, recorded at three different heights, at a voltage of $-0.34 \mathrm{eV}$ (cf. Fig. 6). The experimental image at $Z=5.5 \AA$ was recorded at $U=-0.01 \mathrm{eV}$ (see text). interpretation of the orientation of each molecule.

In addition to the distance dependence, the quality and resolution of the STM topographies depend on the tip orbital. The traditional approach by Tersoff and Hamann ${ }^{35}$ simply assumes an $s$-like orbital, which probes the value of the sample wave function at the position of the tip apex. In experiment, the nature of this orbital is often not known. In particular, a transition-metal tip might be characterized by $d$-like apex orbitals, of which the $\left(3 z^{2}-r^{2}\right)$ orbital would stick out farthest toward the sample and, thus, yield maximum tunneling current. Another possibility to change the nature of the tip is its decoration by adsorbates, e.g., by molecules that are picked up from the sample. This might result in $p$-like orbitals, of which the $p_{z}$ orbital sticks out and would dominate the current. By including the corresponding operators in our approach (see Sec. III), we obtain the images shown in the right column of Fig. 6. The $d_{3 z^{2}-r^{2}}$ orbital yields significantly higher contrast than the $s$ orbital, while again suffering from the loss of resolution when the tip is pulled off. Simply speaking, at a given tip height (e.g., $8 \AA$ ), the $d_{3 z^{2}-r^{2}}$ tip shows features which the $s$ tip would show at 1-2 $\AA$ closer distance (i.e., 6-7 $\AA$ ). We believe that this qualitative difference is not caused by the different currents achieved by $s$ and $d$ tips, but rather by the composition of the sample wave functions of various Fourier components.

The reason for the distance dependence of the images resides in the different spatial decay character of the various $\mathbf{G}$ components of the wave functions. As can be seen from Eq. (7), large $\mathbf{G}$ causes a large value of $\lambda$ and, correspondingly, fast decay of $\exp \left[-\lambda\left(z-z_{0}\right)\right]$. This leads to a fast loss of contrast when the tip-sample distance is increased, since at larger heights the tunneling current is dominated by the small-G components. The large-G components, which are responsible for structural details on short length scales, die off much more rapidly. This behavior also depends on the tip orbital: a tip orbital of higher angular momentum (i.e., $p$ or d) requires to take derivatives of $a_{\mathbf{G}}(z)$ with respect to $z$, which brings in additional factor(s) of $\lambda$ [cf. Eqs. (7) and (8)]. Since $\lambda$ increases with $|\mathbf{G}|$, the derivatives favor the larger-G components, thus enhancing the spatial contrast of the tunneling images as compared to $s$-tip images.

Direct comparison of our calculated images with measured data is demonstrated in Fig. 8, in which simulated and experimental data recorded at a voltage of $-0.34 \mathrm{eV}$ are displayed. As mentioned before, in the experiment, the tip was approached until mechanical contact with the molecule was reached, which we estimate to happen at a tip-molecule distance of about $2.5 \AA$ (i.e., at a height of about $5.5 \AA$ above the silver substrate), thus enabling us to measure the tipsurface distance. The corresponding currents amount to $10 \mathrm{nA}(0.1 \mathrm{nA})$ for $Z=7.5 \AA(9.5 \AA)$, thus confirming our estimate of a current drop of about one magnitude per $1 \AA$ distance increase. At the closest distance, however, the voltage had to be reduced to $-0.01 \mathrm{eV}$ to avoid electrical breakthrough. The data confirm our conclusions about the fast loss in spatial resolution when the tip-sample distance is increased.

In Fig. 8, it is also apparent that our experimental images are best described by calculations using an $s$ orbital. In ex- 
periment, images of this character are always obtained if the tip is prepared by dipping it carefully into the $\mathrm{Ag}$ surface. In spectroscopy, such tips reproducibly yield a clear step at the onset of the $\operatorname{Ag}(111)$ surface state, with very little additional structure due to tip artifacts. ${ }^{20}$

It is notable that the agreement between the s-tip simulations and the experiments in Fig. 8 deteriorates for close tip-sample distances. The experimental image taken at $5.5 \AA$ appears much more blurred than the theoretical one, while the essential structures in the images agree quite well; in particular, the evolution of the spatial intensity distribution when approaching from 7.5 to $5.5 \AA$ tip-sample distance is accurately described by the calculation. From our experiments, we know that the scanning conditions for the $5.5 \AA$ image are very close to the threshold where abrupt changes in the structure of the tip-molecule junction can be detected as sharp steps in the tunneling current. It is not at all improbable that gradual changes in the junction structure take place even before this threshold. This suggests that the blurred nature of the upper experimental image in Fig. 8 is, in fact, due to a transient distortion of the molecular film as the tip scans the surface. Such effects are not included in the current STM simulation. However, it has been shown theoretically that approximately $2 \AA$ before contact, mechanical distortions in the STM junction are to be expected. ${ }^{53}$

In Ref. 20, a comparison between theoretical STM images of the PTCDA: $\operatorname{Ag}(111)$ system, simulated within the same approach as taken here, and experimental data has been presented. In Figs. 3(a)-3(c) of Ref. 20, we have reported images recorded at tip heights between 7 and $9 \AA$, which show much clearer contrast than the two corresponding images in Fig. 8 of the present paper. On the basis of the simulation, the images in Fig. 3 of Ref. 20 have been assigned to a $d$ tip. Such images with apparent characteristics of tip orbitals of higher angular momentum are regularly observed if the STM tip is functionalized by a molecule. For example, after picking up a PTCDA molecule from the surface by controlled atomic manipulation, we very often observe contrasts as shown in Fig. 3(a)-3(c) of Ref. 20. This effect might indicate that a $p$-like orbital of the tip molecule is responsible for the tunneling current. However, the improved resolution might as well be unrelated to the symmetry of the tip orbital: We have mentioned above that in the simulation, a $d$ tip yields the resolution which an $s$ tip would yield if scanned 1-2 $\AA$ closer to the surface, while qualitatively there is no real difference between the types of image contrasts obtained with the two orbitals. It is, therefore, conceivable that the molecule under the tip reduces the electronically relevant tipsample distance without leading to the strong tip-molecule interaction which is expected between the metallic tip and the adsorbed layer and which, as we have argued, blurs the STM image. In this geometry, even an $s$ orbital at the apex of the functionalized tip could lead to the enhanced resolution observed in Ref. 20. In this scenario, the tip-suspended molecule plays the role of a spacer which reduces the mechanical tip-sample interaction while bringing the electronically relevant apex close to the sample.

\section{B. Topographic images at various voltages}

At close distance (5-6 $⿱$ ) ), the images shown in Fig. 6 are clearly identified as resulting from the LUMO orbital. This
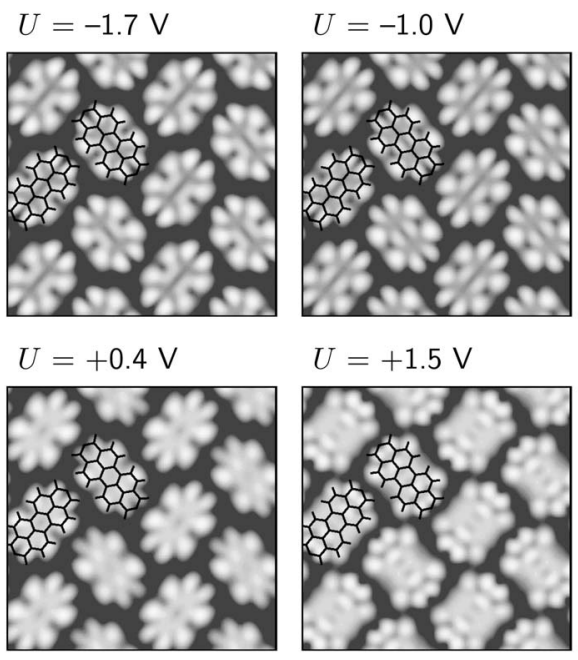

FIG. 9. Calculated topographical constant-current images of a monolayer of PTCDA on the $\mathrm{Ag}(111)$ surface, recorded at four different voltages (cf. Fig. 2). Tip orbital: $d_{3 z^{2}-r^{2}}$. Tip height: $7 \AA$.

behavior depends, however, on the tunneling voltage. Note that only states between the Fermi level and the chosen tunneling voltage contribute to the current. For the voltage of $U=-1 \mathrm{~V}$ (Fig. 6), the overwhelming contribution to the tunneling comes from the former LUMO state, thus explaining the close resemblance to the wave function shown in Fig. 3. At voltages below $U \approx-1.3 \mathrm{~V}$ or above $U \approx+0.3 \mathrm{~V}$, other molecular states contribute to the current, as well (cf. Fig. 2), thus changing the images qualitatively. As illustration, Fig. 9 displays topographical images at four different voltages, corresponding to the voltages indicated in Fig. 2(a). At $U=-1.7 \mathrm{~V}$, the tunneling current results from a superposition of the HOMO and LUMO, but is still dominated by the LUMO (cf. the relative weights of the peaks in Fig. 2). The continued dominance of the LUMO is caused by the higher energy of the LUMO, leading to significantly smaller $\lambda$ in Eq. (7). In essence, the HOMO state decays much more rapidly into the vacuum than the LUMO, which, therefore, contributes more strongly to the current, even at $U=-1.7 \mathrm{eV}$. Consequently, the topographic image at $U=-1.7 \mathrm{~V}$ is very similar to the one at $U=-1 \mathrm{~V}$. Nonetheless, close inspection reveals that the strong minimum of the current at $\mathrm{C}-\mathrm{C}$ bond 2 (see Fig. 4), as observed at $-1 \mathrm{~V}$, appears somewhat filled at $-1.7 \mathrm{eV}$. This results from the HOMO state which has a lobe at that bond, as opposed to the LUMO state which has a node there. The combination of both features leads to the "Vshaped" structures opening toward the sides of the molecule. These features have been observed in Fig. 3(b) of Ref. 20, with a functionalized tip and at a tip-sample distance of $6.8 \AA$. Incidentally, the relatively strong decay of the HOMO wave function away from the surface also explains the difficulty of recording spectra of the HOMO with a nonfunctionalized tip [cf. Fig. 2(c) and Ref. 20].

At small positive voltage of $+0.4 \mathrm{~V}$, the topographic image is again dominated by the LUMO structure, yielding images very similar to the ones recorded at $-1.0 \mathrm{~V}$. At larger positive voltage of $+1.6 \mathrm{~V}$, finally, contributions from the $\mathrm{LUMO}+1$ and LUMO +2 states dominate the image, as can 

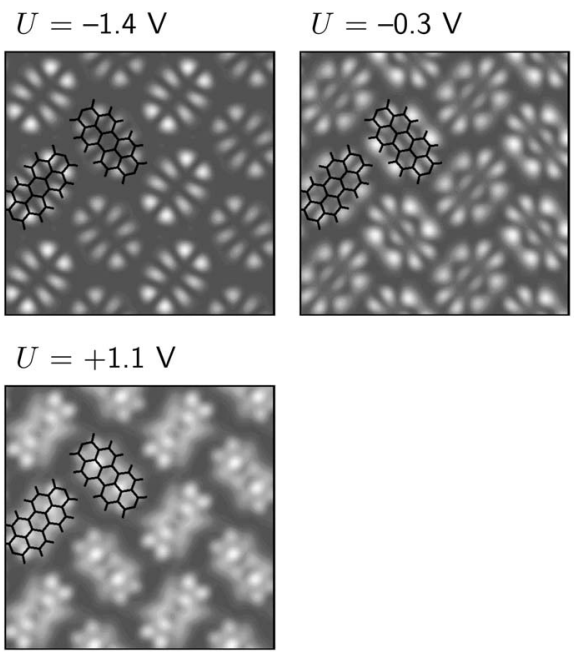

FIG. 10. Calculated spectroscopic images of a monolayer of PTCDA on the $\operatorname{Ag}(111)$ surface, recorded at constant height of $7 \AA$ at three different voltages (cf. Fig. 2), showing the HOMO state (at $-1.4 \mathrm{eV}$ ), LUMO state (at $-0.3 \mathrm{eV}$ ), and a superposition of $\mathrm{LUMO}+1$ and $\mathrm{LUMO}+2($ at $+1.1 \mathrm{eV})$. Tip orbital: $d_{3 z^{2}-r^{2}}$.

clearly be seen from the large number of current maxima found at each molecule. Note that the LUMO+1 and LUMO +2 states are so high in energy (i.e., slowly decaying into vacuum) that their contribution to the tunneling current massively dominates the image. Since the energetic difference between the LUMO+1 and LUMO+2 states is much smaller than their broadening due to coupling to the substrate, most features at tunneling voltages above $U \sim 0.5 \mathrm{eV}$ result from both states simultaneously. Due to the quite different wave functions of both states (see Fig. 3), the spatial resolution of STM and STS images at positive voltage is quite limited.

\section{Spectroscopic images}

The individual identification of molecular states is most easily achieved in the spectroscopic imaging mode, in which the energy summation in Eq. (1) is replaced by a simple energy evaluation at the desired voltage [see Eq. (8)]. Experimentally, this corresponds to taking the derivative of the current with respect to voltage, $\partial I / \partial U$. Calculated data, obtained at constant height, are shown in Fig. 10. At voltages of $-1.4,-0.1$, and $+1.3 \mathrm{~V}$, the resulting images directly show the exclusive contribution of the HOMO, LUMO, and LUMO+1/LUMO+2 states (cf. Fig. 2).

A comparison of our calculated STS images with experimental data is shown in Fig. 11. It is obvious that, once more, $d$-tip images show a better resolution than $s$-tip images.

Interestingly, at the present height of $9 \AA$, the HOMO state shows much better spatial resolution than the LUMO and $\mathrm{LUMO}+1 / \mathrm{LUMO}+2$ states. This is mostly related to the signs of the lobes of the HOMO wave functions [cf. Fig. 3(a)], yielding nodal planes between the lobes which extend into vacuum, thus suppressing the tunneling current. ${ }^{39}$ Correspondingly, sharply resolved features with exactly vanish-

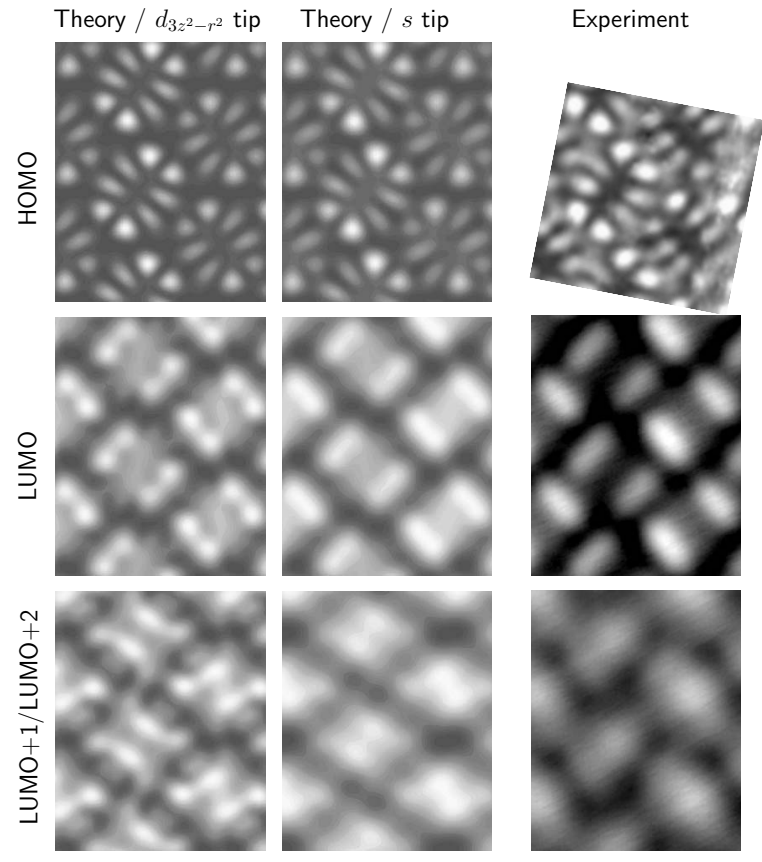

FIG. 11. Calculated and measured spectroscopic images recorded at the positions of the HOMO, LUMO, and LUMO $+1 /$ LUMO +2 states at a height of $9 \AA$ above the $\operatorname{Ag}(111)$ surface (cf. Fig. 6). The measured HOMO STS image was recorded at a height of $7.5 \AA$. The voltages correspond to $-1.4 \mathrm{eV}(-0.1 \mathrm{eV}$, $+1.1 \mathrm{eV})$ in the calculated images, compared to $-1.6 \mathrm{eV}(-0.16 \mathrm{eV}$, $0.73 \mathrm{eV})$ in experiment (see text). Theory and experiment are presented at different voltages because of deviations in the orbital energies between the two.

ing current in between appear inside each molecule.

In the case of the LUMO state, the lobes have the same sign along the long axis of the molecule [cf. Fig. 3(b)], causing constructive superposition of wave-function amplitude along the molecule at larger tip-sample distance. Consequently, all structural information along the long axis of the molecule is lost in tunneling data at larger height. Perpendicular to the long axis of the molecule, destructive interference takes place, as well, resulting from positive, negative, positive, and negative contributions to the wave function (cf. Fig. 3), leaving only the side lobes as dominant contribution at larger height. The structure inside the image of each molecule (clear nodal structure of the HOMO, no internal structure for the LUMO) is, thus, a direct consequence of the composition of the molecular state from positive and negative lobes, as can be easily verified by simple superposition of spherical waves (not shown here).

In the case of the LUMO+1 and LUMO+2 states, their very similar energies and significant broadening pose a similar problem concerning spatial resolution, as already discussed in Sec. IV B. The two states overlap and, thus, contribute to the STS image simultaneously. Since both states have quite different wave functions, STS images at positive voltage consist of up to 16 lobes (cf. Fig. 3) which merge into a quite structureless feature in the center of the molecule, accompanied by distinct lobes near the oxygen end groups (see Fig. 10 at $+1.1 \mathrm{eV}$ at a height of $7 \AA$ above Ag). At larger height of $9 \AA$ above $\mathrm{Ag}$, superposition behavior 
similar to that of the LUMO state causes further loss of details and merging of features from the two states, yielding quite blurred images (see Fig. 11). In particular, in the experimental image, there is basically no atomic resolution any more at positive voltage. Spatial resolution for the LUMO, LUMO + 1, and LUMO+2 states is further reduced due to their significant interaction with the substrate, which leads to hybridization with substrate states.

\section{Differences between molecules A and B}

As is to be expected from the similar local adsorption geometries, the electronic properties and STM images of both types of molecules (A and B) are similar. Nevertheless, there are characteristic differences, caused by the distortion of the surface unit cell and the different alignments of the molecules with the substrate lattice (cf. the discussion of Fig. 2). In the case of the LUMO state, the two molecules have different spectral weights below $E_{F}$, leading to different tunneling currents and concomitantly different brightness in the STM topography (see Fig. 6). In the case of the HOMO, the spectral weight of both molecules is about the same, but is found at slightly different energies. Concomitantly, in the STS images, the two molecules appear at slightly different voltages. This can be seen in the upper left panel of Fig. 10 (at $-1.4 \mathrm{eV}$ ), which has been calculated at a voltage at which molecule B has larger amplitude than molecule A. At slightly different voltage, the amplitude ratio would be inverted. Similar effects are visible at the other voltages discussed in Fig. 10. When tuning the voltage, the brightness ratio between molecules A and B will change. For all states, slight modification $(<0.1 \mathrm{eV})$ of the tunneling voltage already allows one to switch the STS contribution of molecule A or B on and off, relating to their slightly different energies. The contrast difference between A and B molecules is clearly observed in experimental STM topographies and STS images (cf. Figs. 8 and 11).

As discussed before, these effects combine the influence of molecule-substrate interaction [depending on the alignment of each molecule with the $\operatorname{Ag}(111)$ lattice] and intermolecular interaction (depending on the hydrogen bonds). Owing to the subtle character of these effects and to possible additional many-body effects to the spectra neglected in our calculations, it is not surprising that the on and/or off switching of the molecules occurs at slightly different voltages in our calculations as compared to the measurements.

\section{CONCLUSIONS}

In this paper, we have reported a comprehensive DFT study of the adsorption system PTCDA:Ag(111), an example of a weakly chemisorbed $\pi$-conjugated molecule on a metal surface. Detailed results concerning the intralayer geometry and corresponding electronic structure have been obtained. By comparison to the very broad experimental database available for this model system, the virtues and deficiencies of the chosen density-functional approach have been identified. The most severe remaining problems concern the absolute value of the adsorption height and the adsorption energies, which we attribute to insufficiencies of the available DFT exchange-correlation functionals, which do not include van der Waals interactions in a systematic way. For the time being, we have restricted ourselves to the local-density approximation since it yields more realistic molecule-substrate equilibrium distances than all of the available gradient functionals.

These problems notwithstanding, the DFT-LDA approach to PTCDA: $\operatorname{Ag}(111)$ yields the correct adsorption site, an overall electronic structure and molecular distortions in nearly quantitative agreement with experiment, a site specificity of the electronic structure which is qualitatively correct (including valuable hints concerning the role of intermolecular and interfacial interactions in this context), a correct description of charge transfer from the metal to the molecule, a scenario concerning the interplay of two bonding mechanisms which is in good agreement with all available experimental data, and very realistic scanning tunneling microscopy and spectroscopy simulations.

The results reported here substantially improve our understanding of the PTCDA:Ag(111) interface by providing a model which integrates the available experimental data into a coherent picture. We believe, however, that the present study also points beyond PTCDA: $\operatorname{Ag}(111)$ by exemplifying what DFT can currently achieve for comparable adsorption systems and interfaces. In this context, the material system PTCDA: $\operatorname{Ag}(111)$ with its broad database is an ideal test case for a comprehensive evaluation of DFT.

With the increased interest in organic and molecular electronics, in the future the demand for accurate calculations for similar interfaces as the one treated here will certainly rise. Because of their large size and complexity, there is currently no computational method which fulfills all requirements of these object system in an ideal way. However, our study shows that DFT in the LDA approximation, while certainly having serious problems with some aspects of the task, is still a relatively efficient method to arrive at reliable predictions. However, in the face of the potential problems, the results of DFT-LDA for these systems should always be validated independently.

\section{ACKNOWLEDGMENTS}

We are grateful for computational resources provided by the John von Neumann Institut für Computing (NIC) at the Forschungszentrum Jülich. 
*Corresponding author; michael.rohlfing@uni-osnabrueck.de

${ }^{1}$ M. Jung, U. Baston, G. Schnitzler, M. Kaiser, J. Papst, T. Porwol, H. J. Freund, and E. Umbach, J. Mol. Struct. 293, 239 (1993).

${ }^{2}$ J. Taborski, P. Väterlein, H. Dietz, U. Zimmermann, and E. Umbach, J. Electron Spectrosc. Relat. Phenom. 75, 129 (1995).

${ }^{3}$ C. Seidel, C. Awater, X. D. Liu, R. Ellerbrake, and H. Fuchs, Surf. Sci. 371, 123 (1997).

${ }^{4}$ E. Umbach, K. Glöckler, and M. Sokolowski, Surf. Sci. 402, 20 (1998).

${ }^{5}$ K. Glöckler, C. Seidel, A. Soukopp, M. Sokolowski, E. Umbach, M. Böhringer, R. Berndt, and W.-D. Schneider, Surf. Sci. 405, 1 (1998).

${ }^{6}$ B. Krause, A. C. Dür, K. A. Ritley, F. Schreiber, H. Dosch, and D. Smilgies, Appl. Surf. Sci. 175, 332 (2001).

${ }^{7}$ B. Krause, A. C. Dürr, K. Ritley, F. Schreiber, H. Dosch, and D. Smilgies, Phys. Rev. B 66, 235404 (2002).

${ }^{8}$ F. S. Tautz, M. Eremtchenko, J. A. Schaefer, M. Sokolowski, V. Shklover, and E. Umbach, Phys. Rev. B 65, 125405 (2002).

${ }^{9}$ F. S. Tautz, M. Eremtchenko, J. A. Schaefer, M. Sokolowski, V. Shklover, K. Glöckler, and E. Umbach, Surf. Sci. 502, 176 (2002).

${ }^{10}$ S. Picozzi, A. Pecchia, M. Gheorghe, A. Di Carlo, P. Lugli, B. Delley, and M. Elstner, Phys. Rev. B 68, 195309 (2003).

${ }^{11}$ M. Eremtchenko, J. A. Schaefer, and F. S. Tautz, Nature (London) 425, 602 (2003).

${ }^{12}$ V. Wagner, T. Muck, J. Geurts, M. Schneider, and E. Umbach, Appl. Surf. Sci. 212-213, 520 (2003).

${ }^{13}$ L. Kilian, E. Umbach, and M. Sokolowski, Surf. Sci. 573, 359 (2004).

${ }^{14}$ M. Eremtchenko, D. Bauer, J. A. Schaefer, and F. S. Tautz, New J. Phys. 6, 4 (2004).

${ }^{15}$ A. Hauschild, K. Karki, B. C. C. Cowie, M. Rohlfing, F. S. Tautz, and M. Sokolowski, Phys. Rev. Lett. 94, 036106 (2005).

${ }^{16}$ S. Berger, K. Heimer, H. G. Mack, and C. Ziegler, Appl. Surf. Sci. 252, 81 (2005).

${ }^{17}$ R. Rurali, N. Lorente, and P. Ordejon, Phys. Rev. Lett. 95, 209601 (2005)

${ }^{18}$ A. Hauschild, K. Karki, B. C. C. Cowie, M. Rohlfing, F. S. Tautz, and M. Sokolowski, Phys. Rev. Lett. 95, 209602 (2005).

${ }^{19}$ D. Braun, A. Schirmeisen, and H. Fuchs, Surf. Sci. 575, 3 (2005).

${ }^{20}$ A. Kraft, R. Temirov, S. K. M. Henze, S. Soubatch, M. Rohlfing, and F. S. Tautz, Phys. Rev. B 74, 041402(R) (2006).

${ }^{21}$ Y. Zou, L. Kilian, A. Schöll, Th. Schmidt, R. Fink, and E. Umbach, Surf. Sci. 600, 1240 (2006).

${ }^{22}$ R. Temirov, S. Soubatch, A. Luican, and F. S. Tautz, Nature (London) 444, 350 (2006).

${ }^{23}$ S. X. Du, H. J. Gao, C. Seidel, L. Tsetseris, W. Ji, H. Kopf, L. F. Chi, H. Fuchs, S. J. Pennycook, and S. T. Pantelides, Phys. Rev. Lett. 97, 156105 (2006).

${ }^{24}$ S. K. M. Henze, O. Bauer, T.-L. Lee, M. Sokolowski, and F. S. Tautz, Surf. Sci. 601, 1566 (2007).

${ }^{25}$ L. W. Bruch, M. W. Cole, and E. Zaremba, Physical Adsorption: Forces and Phenomena (Oxford Science, Oxford, 1997).

${ }^{26}$ M.-C. Desjonqueres and D. Spanjaard, Concepts in Surface Science, 2nd ed. (Springer, Berlin, 1996).

${ }^{27}$ A. Liebsch, Electronic Excitations at Metal Surfaces (Plenum,
New York, 1997).

${ }^{28}$ J. F. Dobson and J. Wang, Phys. Rev. Lett. 82, 2123 (1999).

${ }^{29}$ M. Dion, H. Rydberg, E. Schröder, D. C. Langreth, and B. I. Lundqvist, Phys. Rev. Lett. 92, 246401 (2004).

${ }^{30}$ S. D. Chakarova-Käck, E. Schröder, B. I. Lundqvist, and D. C. Langreth, Phys. Rev. Lett. 96, 146107 (2006).

${ }^{31}$ D. M. Ceperley and B. J. Alder, Phys. Rev. Lett. 45, 566 (1980).

${ }^{32}$ J. P. Perdew, K. Burke, and M. Ernzerhof, Phys. Rev. Lett. 77, 3865 (1996).

${ }^{33}$ J. P. Perdew, J. A. Chevary, S. H. Vosko, K. A. Jackson, M. R. Pederson, D. J. Singh, and C. Fiolhais, Phys. Rev. B 46, 6671 (1992).

${ }^{34}$ P. S. Bagus, V. Staemmler, and C. Woll, Phys. Rev. Lett. 89, 096104 (2002).

${ }^{35}$ J. Tersoff and D. R. Hamann, Phys. Rev. B 31, 805 (1985).

${ }^{36}$ C. J. Chen, Phys. Rev. Lett. 65, 448 (1990).

${ }^{37}$ W. Sacks, S. Gauthier, S. Rousset, J. Klein, and M. A. Esrick, Phys. Rev. B 36, 961 (1987).

${ }^{38}$ S. Heinze, S. Blugel, R. Pascal, M. Bode, and R. Wiesendanger, Phys. Rev. B 58, 16432 (1998).

${ }^{39}$ J. K. Garleff, M. Wenderoth, K. Sauthoff, R. G. Ulbrich, and M. Rohlfing, Phys. Rev. B 70, 245424 (2004).

${ }^{40}$ O. Paz, I. Brihuega, J. M. Gomez-Rodriguez, and J. M. Soler, Phys. Rev. Lett. 94, 056103 (2005).

${ }^{41}$ For details about the SIESTA package, please refer to http:// www.uam.es/departamentos/ciencias/fismateriac/siesta/

${ }^{42}$ P. Ordejon, E. Artacho, and J. M. Soler, Phys. Rev. B 53, R10441 (1996).

${ }^{43}$ J. M. Soler, E. Artacho, J. D. Gale, A. Garcia, J. Junquera, P. Ordejon, and D. Sanchez-Portal, J. Phys.: Condens. Matter 14, 2745 (2002).

${ }^{44}$ A. J. Lovinger, S. R. Forrest, M. L. Kaplan, P. H. Schmidt, and T. Venkatesan, J. Appl. Phys. 55, 476 (1984).

${ }^{45}$ M. Möbus, N. Karl, and T. Kobayashi, J. Cryst. Growth 116, 495 (1992).

${ }^{46}$ T. Ogawa, K. Kuwamoto, S. Isoda, T. Kobayashi, and N. Karl, Acta Crystallogr., Sect. B: Struct. Sci. 55, 123 (1999).

${ }^{47}$ N. Dori, M. Menon, L. Kilian, M. Sokolowski, L. Kronik, and E. Umbach, Phys. Rev. B 73, 195208 (2006).

${ }^{48}$ A. Gerlach, S. Sellner, F. Schreiber, N. Koch, and J. Zegenhagen, Phys. Rev. B 75, 045401 (2007).

${ }^{49}$ O. Bauer, S. Soubatch, M. Sokolowski, and F. S. Tautz (unpublished).

${ }^{50}$ L. Kilian, A. Hauschild, R. Temirov, S. Soubatch, A. Schöll, A. Bendounan, F. Reinert, F. S. Tautz, M. Sokolowski, and E. Umbach (unpublished).

${ }^{51}$ A slightly different analysis would be obtained if the potential were nonconstant. The main effect, however, would just be some slowing down of the wave-function decay into the vacuum, hardly affecting the tunneling images.

${ }^{52}$ The reliability of the band-structure energies and of the wave functions are lost as one approaches the vacuum level; consequently, our approach is only valid for voltages smaller than the work function of the surface.

${ }^{53}$ L. Limot, J. Kröger, R. Berndt, A. Garcia-Lekue, and W. A. Hofer, Phys. Rev. Lett. 94, 126102 (2005). 